\title{
A Model for Relative Permeabilities Under Gas Liberation or Condensate Precipitation in Porous Medium
}

\author{
Al-Masri, Wael; Shapiro, Alexander
}

Published in:

Transport in Porous Media

Link to article, DOI:

10.1007/s11242-020-01496-9

Publication date:

2021

Document Version

Peer reviewed version

Link back to DTU Orbit

Citation (APA):

Al-Masri, W., \& Shapiro, A. (2021). A Model for Relative Permeabilities Under Gas Liberation or Condensate Precipitation in Porous Medium. Transport in Porous Media, 136, 43-63 . https://doi.org/10.1007/s11242-02001496-9

\section{General rights}

Copyright and moral rights for the publications made accessible in the public portal are retained by the authors and/or other copyright owners and it is a condition of accessing publications that users recognise and abide by the legal requirements associated with these rights.

- Users may download and print one copy of any publication from the public portal for the purpose of private study or research.

- You may not further distribute the material or use it for any profit-making activity or commercial gain

- You may freely distribute the URL identifying the publication in the public portal

If you believe that this document breaches copyright please contact us providing details, and we will remove access to the work immediately and investigate your claim 


\title{
A Model for Relative Permeabilities Under Gas Liberation or Condensate Precipitation in Porous Medium
}

\author{
Wael Al-Masri ${ }^{1}$, Alexander Shapiro ${ }^{1}$
}

Keywords: Gas liberation, Condensate precipitation, Relative permeability, Microscopic model

\begin{abstract}
We derive a model for relative permeabilities under two-phase flows in porous media, where the phases may partly mix with each other, and one of the phases may be immobile. Particular cases are the bubble formation in an oil reservoir when the pressure falls below the bubble point, or, similarly, condensation and droplet precipitation in a gas-condensate reservoir. The dependencies for the relative permeabilities on the saturation are derived based on a pore-level model of the porous medium, represented as a capillary network. Distribution of the bubbles or droplets in the network is computed with the application of a method similar to the fundamental statistical physics. Model formula for the conductivities of single capillaries, depending on the numbers of bubbles or droplets in them, are converted to the relative permeabilities of the whole lattice by application of the effective medium formalism. We establish universal correlations between the micro-characteristics of the porous medium, constituting our model, and the parameters in the Corey-Brooks dependencies for relative permeabilities: exponents and limiting saturations. In this way, it is also possible to extend the standard Corey-Brooks formula onto the saturation ranges where one of the phases is immobile. A comparison with the available experimental data indicates the good performance of the model. The experimental data may be fitted by variation of a single parameter, and the data from similar rocks have similar parameter values, which indicates the physical soundness of the model.
\end{abstract}

\section{Article Highlights}

- A novel stochastic model for relative permeabilities under gas liberation or condensate precipitation has been developed

- The model matches available experimental data with the only adjustment parameter

- The parameters of the traditional Corey model for relative permeabilities are matched with micro-characteristics of the porous medium.

凶ash@kt.dtu.dk

${ }^{1}$ Technical University of Denmark, Department of Chemical and Biochemical Engineering, 2800 Kgs. Lyngby, Denmark. 


\section{List of designations}

\begin{tabular}{|c|c|}
\hline$b, d$ & The functions of the coordinate number determining the critical saturation \\
\hline$c_{i}$ & The number of capillaries containing $i$ bubbles \\
\hline$C$ & The number of combinations \\
\hline$D$ & The number of arrangements \\
\hline$f_{o}, f_{g}$ & The fractional flow threshold for oil and gas-phase respectively \\
\hline$f$ & The fraction of bubbles/droplets to the maximum capacity of the capillary \\
\hline$F$ & The conductivity distribution function \\
\hline$g^{0}$ & The maximum conductivity \\
\hline$G_{1}, G_{2}$ & The Lagrange constraints \\
\hline$g$ & The conductivity of a single capillary \\
\hline$k_{r}$ & The relative permeability \\
\hline$L$ & The length of a capillary \\
\hline$l, t$ & $\begin{array}{l}\text { The functions of the coordinate number determining the Brooks-Corey } \\
\text { exponents }\end{array}$ \\
\hline$m_{o}, m_{g}$ & The flow thresholds for oil and gas phases, respectively \\
\hline$M$ & The total number of bubbles \\
\hline$m$ & The number of bubbles in a capillary \\
\hline$N$ & The total number of capillaries \\
\hline$n$ & The Brooks-Corey exponent \\
\hline$P$ & The exponents defining the shapes of the conductivity dependences \\
\hline$r$ & The capillary radius \\
\hline$S$ & The saturation \\
\hline$v$ & The volume \\
\hline$x_{i}$ & The fraction of the capillaries containing $i$ bubbles \\
\hline$Z$ & The coordination number \\
\hline$\alpha$ & The shape factor \\
\hline$\beta_{i}, \delta_{i}$ & The parameters in the dependencies for the critical saturation \\
\hline$\Lambda_{1}, \Lambda_{2}$ & The Lagrange multipliers \\
\hline $\mathcal{L}$ & The Lagrangian \\
\hline$\lambda_{i}, \tau_{i}$ & The parameters in the dependencies for the Brooks-Corey exponents \\
\hline
\end{tabular}

\section{Subscripts}

\begin{tabular}{|c|l|}
\hline$b$ & Bubble \\
\hline$c$ & Capillary \\
\hline$c r$ & Critical \\
\hline$e$ & Effective \\
\hline$g$ & Gas \\
\hline$M$ & Maximum \\
\hline$o$ & Oil \\
\hline
\end{tabular}




\section{Introduction}

Relative permeabilities are a critical input for models and simulations used for describing the two-phase and multiphase flows in porous media on the basis of the Buckley-Leverett or a similar model. In particular, reliable relative permeability data are essential for evaluating and optimizing production from petroleum reservoirs (Dake, 1994).

In order to apply the relative permeabilities in various reservoir simulators, their dependencies on phase saturations are required. Commonly, the empirical Corey-Brooks formula is applied (Brooks \& Corey, 1964), in the simplified form of the power-law. Other formulae have also been suggested (Chierici, 1984; Corey \& Rathjens, 1956).

For the two-phase flows, the empirical formulae for relative permeabilities are commonly restricted onto the saturation interval between the limiting saturations where both phases are mobile. Outside this interval, the Corey-Brooks dependencies cannot be applied, since the values become indefinite or meaningless. This does not pose any serious problem for immiscible phases (like oil and water under waterflooding or a water-based method of enhanced oil recovery) since for such flows; the saturations very rarely become smaller than the irreducible or residual values. However, for the partly miscible flows, like, e.g., gas-oil flows where gas may dissolve in oil or be liberated from it, this may be a severe limitation.

Consider, for example, the production of an oil reservoir by depletion. When the pressure falls below a bubble point, the gas may be liberated in the form of separate bubbles in the porous space (Dake, 1994). The bubbles form an immobile gas phase, which plugs the separate paths for oil, and the relative permeability for oil is correspondingly reduced. Similarly, if the pressure falls below the dew point underproduction of a gas-condensate reservoir, the precipitated condensate droplets may form an immobile liquid phase plugging the gas flow (Kalla et al., 2014). Reduction of permeability may be rather significant (Pope et al., 1998) and should somehow be accounted for in the models for relative permeabilities. However, to the best of our knowledge, there has been a scarcity of studies conducted with regards to relative permeabilities for such cases where phase transitions (condensation and evaporation) may take place.

Another challenge is to relate the dependencies for relative permeabilities to the structure of the porous space and peculiarities of the phase distribution in it. One approach for this is the application of the percolation theory in the form of an effective medium (Kirkpatrick, 1973b), r-chains (Selyakov \& Kadet, 1996), and others. Sahimi (2011), Ghanbarian, Hunt, Skinner, \& Ewing, (2015); Hunt, Ewing, \& Ghanbarian, (2014), and Bedrikovetsky (1993) have given thorough literature reviews. Few works are related to the application of a similar process of drying in porous media, but not for calculation of the relative permeabilities (Prat, 2002; I N Tsimpanogiannis, Yortsos, Poulou, Kanellopoulos, \& Stubos, 1999; Woo, 2019). An alternative approach to obtaining the relative permeabilities is direct modeling of the pore space or its approximation by a network model, and numerical computation of the relative permeabilities based on the hydrodynamic rules (Blunt \& King, 1991; Mogensen \& Stenby, 1998). This approach will not be considered in the present work.

The bond percolation models and other capillary network models usually assume that a singlephase occupies each capillary in the network. They utilize the pore size distribution to count for the effect of pore structure on the effective permeability. However, none of them have considered that condensation may occur in the form of the droplets, and, similarly, evaporation 
will result in the bubbles appearing randomly in the different capillaries of the network. A possibility for the simultaneous presence of the different phases in the same capillary of their simultaneous flow, or for blocking of one or both of them, has not been considered in the literature to a sufficient extent to model the relative permeabilities for the partly miscible flows. Each capillary might contain both phases, and one, or two, or none of them might be mobile, although with restricted mobility. This is the situation that we would like to describe in the present work.

In this study, we investigate the effect of gas liberation and condensate precipitation on the relative permeabilities. The porous medium is modeled by a regular lattice of capillaries (bonds). Random distribution of the bubbles or droplets in the lattice is described by the formalism borrowed from the classical statistical physics (Honerkamp, 2012; Kubo, 1965; Landau \& Lifshits, 1980). An alterantive hypothesis, about a correlated distribution of the bubbles is not studied, so that only the simplest possible model is considered. Upon an assumption about the conductivity of a single capillary containing a certain amount of bubbles or droplets, the theory of effective medium is applied to calculate the conductivity of the whole lattice. Then the conductivity of the lattice is determined from the distribution of the conductivities of the differently plugged lattice bonds. The resulting relative permeabilities are compared with the Brooks-Corey dependencies. Universal and relative simple correlations between the parameters of the two models have been obtained. In this way, the parameters of the Brooks-Corey model (critical saturations and exponents) were correlated with the model microscopic parameters of the porous medium, and these dependencies were extended onto the regions of immobile phases. Sensitivity of the model to the different parameters has been studied. Finally, the model has been compared with the experimental data available in the literature. It has been demonstrated that the model is in agreement with the data; moreover, the data from similar rocks may be approximated on the basis of the similar model parameters. In this way, the physical adequateness of the model has been demonstrated.

\section{Theory}

\subsection{Assumptions of the model}

In this study, we represent the porous medium as a lattice. The lattice contains a large number $N$ of cylindrical capillaries of length $L$ and radius $r$. It is assumed to be regular, with the coordination number $Z$. Such lattices are often considered as models for a porous medium in the percolation and effective medium theories. We consider the problem of percolation by bonds so that the porosity of the sites is neglected.

In the described lattice, consider, as an example, the following isothermal process. Initially, the pressure exceeds the bubble point, and the liquid (oil) saturating the porous medium contains dissolved gas. Then the pressure decreases, and after crossing the bubble point, some portion of the gas liberates from the liquid. We assume that the gas appears in the form of bubbles. The number of bubbles $M$, and therefore, the saturation $S_{g}$ of the gas grows as pressure decreases. The described process serves as an example, and other processes where gas liberation occurs, like an increase of the temperature, or the processes where liquid condensation occurs, like condensate precipitation in a gas-condensate reservoir, may be described in a similar way.

Our goal is to build a model for the evolution of the oil relative permeability under such a process. The model will be based on the following assumptions. 
The bubbles are assumed to appear randomly at the different capillaries, even if these capillaries already contain previously formed bubbles. This is the simplest possible assumption; an alternative assumption about the correlated distribution would be much more difficult to handle. This assumption corresponds to the heterogeneous nucleation of the bubbles on the randomly distributed formation sites. It should be remarked that the concept of the "bubble" is relative in our treatment. It is only used to put some discrete measure on the amount of the liberated gas. In fact, the present treatment may be applied independently of the process (decreasing the pressure, or rising the temperature, or...). However, the uncorrelated appearance of the bubbles is a limitation of the model, as discussed in section 4.

The maximum number of the bubbles in a capillary will be denoted by $m_{M}$. If the volume of a bubble is $v_{b}$ and the volume of a capillary is $v_{c}$, the maximum number of the bubbles in the capillary cannot exceed $v_{c} / v_{b}$. For the sake of simplicity, it may be assumed that $m_{M}=v_{c} / v_{b}$. Otherwise, the shape-factor $\alpha$ could be introduced: $m_{M}=\alpha v_{c} / v_{b}$. If, for example, the radius of a bubble is equal to that of a capillary, and the capillary is cylindrical, then $m_{M}=L / 2 r$, so that $\alpha=2 / 3$. The bubbles may coalesce, which may also change the shape factor.

The gas saturation may be expressed in terms of the total number of bubbles $M$, or of the average number of bubbles in a single capillary $\langle m\rangle$ :

$$
S_{g}=\frac{M}{N m_{M}}=\frac{\langle m\rangle}{m_{M}}
$$

\subsection{Statistical distribution of the bubbles in the capillaries}

Upon the assumptions formulated above, we can define $D\left(N, M, c_{2}, c_{3}, \ldots, c_{m_{M}}\right)$ as the number of arrangements of the bubbles, such that exactly $c_{i}$ capillaries contain $i$ bubbles, where $i=$ $0,1,2, \ldots, m_{M}$. The value of $M$ should be larger than $\sum_{i=2}^{m_{M}} i c_{i}$. The number of capillaries containing exactly one bubble is

$$
c_{1}=M-\sum_{i=2}^{m_{M}} i c_{i}
$$

The number of capillaries with no bubbles in them is

$$
c_{0}=N-c_{1}-\sum_{i=2}^{m_{M}} c_{i}=N-M+\sum_{i=2}^{m_{M}}(i-1) c_{i}
$$

\subsubsection{The case of two bubbles}

For simplicity, let us consider the case where the capillary can contain at most two bubbles: $m_{M}=2$. The number of arrangements to be calculated is then $D\left(N, M, c_{2}\right)$. For each arrangement, the numbers of the capillaries containing one or no bubbles are, according to Eqs. (2) and (3)

$$
c_{1}=M-2 c_{2} ; c_{0}=N-M+c_{2}
$$

In order to calculate $D\left(N, M, c_{2}\right)$, first, we arrange $c_{2}$ capillaries filled by the two bubbles among $N$ possible places. This may be carried out by $C\left(N, c_{2}\right)$ ways, where $C\left(N, c_{2}\right)$ is a combination number: 


$$
C\left(N, c_{2}\right)=\frac{N !}{c_{2} !\left(N-c_{2}\right) !}
$$

Each of the rest $c_{1}=M-2 c_{2}$ bubbles occupies a separate capillary. Although each capillary has a place for two bubbles, we count it just once, since a position of the bubble in the capillary is not fixed. Thus, the distribution of the $c_{1}$ bubbles can be produced by $C\left(N-c_{2}, c_{1}=m-\right.$ $2 c_{2}$ ) ways.

The total number of ways to arrange the bubbles is thus equal to

$$
D\left(N, M, c_{2}\right)=C\left(N, c_{2}\right) C\left(N-c_{2}, c_{1}\right)=\frac{N !}{c_{2} !\left(M-2 c_{2}\right) !\left(N-M+c_{2}\right) !}
$$

The symmetric way to express this quantity is

$$
D\left(N, M, c_{2}\right)=\frac{N !}{c_{2} ! c_{1} ! c_{0} !}
$$

It should be noticed, however, that in this equation, the values of $c_{i}$ are not independent but connected by relations (4), also involving the total number of bubbles $M$.

\subsubsection{The most probable values}

Let us determine the most probable values of $c_{i}$ or, rather, the fractions $x_{i}=c_{i} / N$. The number of pores and bubbles are usually very large. Following the classical approach of statistical mechanics (Kubo, 1965). we substitute factorials by the Stirling approximation:

$$
A ! \approx \sqrt{2 \pi A}\left(\frac{A}{e}\right)^{A}
$$

Then Eq. (6) is after transformations reduced to

$$
D\left(N,\langle m\rangle, x_{2}\right) \approx \frac{1}{2 \pi N} \sqrt{\frac{1}{x_{2}\left(\langle m\rangle-2 x_{2}\right)\left(1-\langle m\rangle+x_{2}\right)}} \times \frac{1}{x_{2}^{N x_{2}}\left(\langle m\rangle-2 x_{2}\right)^{N\left(\langle m\rangle-2 x_{2}\right)}\left(1-\langle m\rangle+x_{2}\right)^{N\left(1-\langle m\rangle+x_{2}\right)}}
$$

Here $\langle m\rangle=M / N$ is the average number of bubbles in each capillary. The values in the denominator are fractions of unity raised to very large powers proportional to $N$. These are the functions with sharp maxima or minima. Therefore, their product $D\left(N,\langle m\rangle, x_{2}\right)$ has a sharp maximum at some $x_{2}$. Deviations from this value of $x_{2}$ are highly improbable. We take the most probable of $x_{2}$, corresponding to the maximum of $D\left(N,\langle m\rangle, x_{2}\right)$, as the real fraction of the capillaries filled by two bubbles.

It is convenient to find the maximum of the logarithm of $D\left(N,\langle m\rangle, x_{2}\right)$, equal to:

$$
\begin{gathered}
\ln D\left(N,\langle m\rangle, x_{2}\right) \approx-\ln (2 \pi N)-\ln \left(x_{2}\left(\langle m\rangle-2 x_{2}\right)\left(1-\langle m\rangle+x_{2}\right)\right) \\
-N\left[x_{2} \ln \left(x_{2}\right)+\left(\langle m\rangle-2 x_{2}\right) \ln \left(\langle m\rangle-2 x_{2}\right)+\left(1-\langle m\rangle+x_{2}\right) \ln \left(1-\langle m\rangle+x_{2}\right)\right]
\end{gathered}
$$

The last three terms are proportional to $N$, while the first two have a lower order with regard to $N$. Hence, in the limit of large $N$, only the last terms matter:

$$
\begin{aligned}
\ln D\left(N,\langle m\rangle, x_{2}\right) & \\
& \approx-N\left(x_{2} \ln \left(x_{2}\right)+\left(\langle m\rangle-2 x_{2}\right) \ln \left(\langle m\rangle-2 x_{2}\right)\right. \\
& \left.+\left(1-\langle m\rangle+x_{2}\right) \ln \left(1-\langle m\rangle+x_{2}\right)\right)
\end{aligned}
$$


Differentiating and finding equating the derivative to zero, we find the most probable value of $x_{2}$ :

$$
x_{2}=\frac{\langle m\rangle}{2}+\frac{1-\sqrt{-3\langle m\rangle^{2}+6\langle m\rangle+1}}{6}
$$

Correspondingly, the values of $x_{1}$ and $x_{0}$ are expressed by:

$$
\begin{gathered}
x_{1}=\frac{\sqrt{-3\langle m\rangle^{2}+6\langle m\rangle+1}-1}{3} \\
x_{0}=1-\frac{\langle m\rangle}{2}+\frac{1-\sqrt{-3\langle m\rangle^{2}+6\langle m\rangle+1}}{6}
\end{gathered}
$$

In the future computations, we will neglect the fact that the values of $x_{i}$ may deviate from the most probable values estimated by eq. (10), (11) and (12) and only use these averages.

\subsubsection{Generalization onto multiple bubbles}

The same procedure, as described in the previous subsection, is applied for the derivation of the expression for the number of arrangements $D\left(N, M, c_{2}, \ldots, c_{m_{M}}\right)$ with an arbitrary value of $m_{M}$. As previously, transfer to the variables $x_{i}=\frac{c_{i}}{N}$, application of the Stirling approximation and neglect of the lower-order terms results in

$$
N^{-1} \ln D\left(N,\langle m\rangle, x_{2}, \ldots, x_{m_{M}}\right) \approx-\ln \left(\prod_{i=0}^{m_{M}}\left(x_{i}\right)^{x_{i}}\right)
$$

The most probable values of $x_{i}$ are found by maximization of $\ln D\left(N,\langle m\rangle, x_{2}, \ldots, x_{m_{M}}\right)$ under conditions Eqs. (2) and (3). This can be achieved by the method of Lagrange multipliers. The Lagrangian function of the problem has the form of

$$
\mathcal{L}\left(x_{0}, \ldots, x_{m_{M}}, \Lambda_{1}, \Lambda_{2}\right)=f\left(x_{0}, \ldots, x_{m_{M}}\right)-\sum_{i=1}^{2} \Lambda_{i} G_{i}\left(x_{0}, \ldots, x_{m_{M}}\right)
$$

Here

$$
\begin{gathered}
f\left(x_{0}, \ldots, x_{m_{M}}\right)=-\ln \left(\prod_{i=0}^{m_{M}}\left(x_{i}\right)^{x_{i}}\right) \\
G_{1}\left(x_{0}, \ldots, x_{m_{M}}\right)=1-\sum_{i=0}^{m_{M}} x_{m_{M}}=0 ; \\
G_{2}\left(x_{0}, \ldots, x_{m_{M}}\right)=\langle m\rangle-\sum_{i=0}^{m_{M}} i x_{m_{M}}=0
\end{gathered}
$$

Functions $G_{1}, G_{2}$ are the transformed equality constraints from Eq. (4); and $\Lambda_{1}, \Lambda_{2}$ are the Lagrange multipliers. Equating $\nabla \mathcal{L}$ to zero reduces the problem of finding the most probable values of $x_{0}, \ldots, x_{m_{M}}$ to solving $m_{M}+3$ equations for $m_{M}+3$ unknowns $x_{m}(m=$ $\left.0, \ldots, m_{M}\right), \Lambda_{i}(i=1, .2)$ : 


$$
\nabla_{x_{0}, \ldots, x_{m_{M}}, \Lambda_{1}, \Lambda_{2}} \mathcal{L}\left(x_{0}, \ldots, x_{m_{M}}, \Lambda_{1}, \Lambda_{2}\right)=0 \leftrightarrow\left\{\begin{array}{c}
\nabla f\left(x_{0}, \ldots, x_{m_{M}}\right)-\sum_{i=1}^{2} \lambda_{i} \nabla G_{i}\left(x_{0}, \ldots, x_{m_{M}}\right)=0 \\
G_{1}\left(x_{0}, \ldots, x_{m_{M}}\right)=G_{2}\left(x_{0}, \ldots, x_{m_{M}}\right)=0
\end{array}\right.
$$

This system may only be solved numerically for each particular value of $m_{M}>2$ and each value of $\langle m\rangle$. The solution depends only on these values. According to Eq. (1), $\langle m\rangle$ is proportional to gas saturation $S_{g}$. Thus, the distribution $x_{0}, \ldots, x_{m_{M}}$ depends on the gas saturation only.

\subsection{Computation of the relative permeabilities}

\subsubsection{The effective medium theory}

In order to estimate relative permeability on the basis of the obtained distribution $x_{2}, \ldots, x_{m}$, we use the effective medium theory (Kirkpatrick, 1973). This theory makes it possible to evaluate effective conductivity $g_{e}$ of an element in a lattice, where the site conductivities are distributed according to some law $F(g)$. Then the conductivity of the whole lattice is approximated by a homogeneous lattice consisting of the elements $g_{e}$. The value of $g_{e}$ is found from a transcendental equation(Bedrikovetsky, 1993).

$$
\int \frac{g_{e}-g}{g+\left(\frac{Z}{2}-1\right) g_{e}} \cdot F(g) \cdot d g=0
$$

In our case $F(g)$ is a discrete distribution. If a capillary contains $m$ bubbles, its hydraulic conductivity to oil will be denoted by $g_{o, m}$. The fraction of such capillaries is the $x_{m}\left(S_{g}\right)$ found in the previous section. Thus, the effective medium equation (16) is reduced to

$$
\sum_{m=0}^{m_{M}} \frac{g_{o, e}-g_{o, m}}{g_{o, m}+\left(\frac{Z}{2}-1\right) g_{o, e}} \cdot x_{m}\left(S_{g}\right)=0
$$

and similarly for gas conductivity

$$
\sum_{m=0}^{m_{M}} \frac{g_{g, e}-g_{g, m}}{g_{g, m}+\left(\frac{Z}{2}-1\right) g_{g, e}} \cdot x_{m}\left(S_{g}\right)=0,
$$

where $g_{g, m}$ are conductivities of the capillaries containing $m$ bubbles.

\subsubsection{The particular formula for pore hydraulic conductivities}

In order to compute the relative permeabilities with the formulated model, particular expressions for the pore conductivities $g_{o, m}, g_{g, m}$ are required.

Assume that the fluid behavior in the capillary is determined by the two flow threshold values, $m_{o}$ and $m_{g}$. If the number of bubbles is smaller than $m_{o}$, the oil in the capillary can flow, probably, with reduced conductivity. Gas starts flowing if the number of bubbles exceeds $m_{g}$. If $m_{o}<m_{g}$, neither fluid flows if the number of bubbles in between the flow thresholds. Otherwise, if $m_{o}>m_{g}$, both fluids can flow between these thresholds. 
The oil conductivities $g_{o, m}$ decreases with the number of bubbles $m$, becoming zero at $m=$ $m_{o}$. Similarly, the gas conductivity $g_{g, m}$ increases with the number of bubbles becoming zero at $m=m_{g}$ and turning into a maximum conductivity $g^{0}$ at $m=m_{M}$. In the following sample computations, we assume the following dependencies:

$$
\begin{gathered}
g_{o, m}=\left\{\begin{array}{c}
g^{0}\left(1-\frac{m}{m_{o}}\right)^{P_{o}}, m \leq m_{o} ; \\
0, m>m_{o}
\end{array}\right. \\
g_{g, m}=\left\{\begin{array}{c}
g^{0}\left(\frac{m-m_{g}}{m_{M}-m_{g}}\right)^{P_{g}}, m \geq m_{g} ; \\
0, \quad m<m_{g}
\end{array}\right.
\end{gathered}
$$

Here $P_{o}, P_{g}$ are exponents defining the shapes of dependencies. It is expected that $P_{g}<1$ and $P_{o}>1$ since the gas may start flowing with a relatively high rate just after the threshold is crossed, while for oil the rates close to the threshold are small; in other words, the dependence of $g_{o}$ on $m$ is likely to be convex, while for the gas, it is concave.

It is convenient to express the conductivity in terms of the fraction $f=\frac{m}{m_{M}}$, rather than of the number of bubbles in the capillary. The fractional thresholds are defined as $f_{o}=\frac{m_{o}}{m_{M}}$ and $f_{g}=$ $\frac{m_{g}}{m_{M}}:$

$$
\begin{aligned}
& \frac{g_{o, m}}{g^{0}}=\left\{\begin{array}{c}
\left(1-\frac{f}{f_{o}}\right)^{P_{o}}, \quad f \leq f_{o} \\
0, \quad f>f_{o}
\end{array}\right. \\
& \frac{g_{g, m}}{g^{0}}=\left\{\begin{array}{cl}
\left(\frac{f-f_{g}}{1-f_{g}}\right)^{P_{g}}, & f \geq f_{g} \\
0, & f<f_{g}
\end{array}\right.
\end{aligned}
$$

With known values $g_{o, m}, g_{g, m}$, equations (17) and (18) are solved for each saturation $S_{g}$, so that the values $g_{o, e}\left(S_{g}\right), g_{g, e}\left(S_{g}\right)$ are determined. Then the relative permeabilities are found as

$$
k_{r o}\left(S_{g}\right)=\frac{g_{o, e}\left(S_{g}\right)}{g_{o, e}(0)} ; k_{r g}\left(S_{g}\right)=\frac{g_{g, e}\left(S_{g}\right)}{g_{g, e}(1)}
$$

Fig. 1 illustrates the steps needed to compute relative permeabilities for both oil and gas phases.

For some values of $S_{g}$, equations (17), (18) do not have a solution between zero and maximum possible conductivity $g^{0}$. The least values of saturation, for which a meaningful solution exists, corresponding to the critical saturations beyond which one of the phases is immobile. 


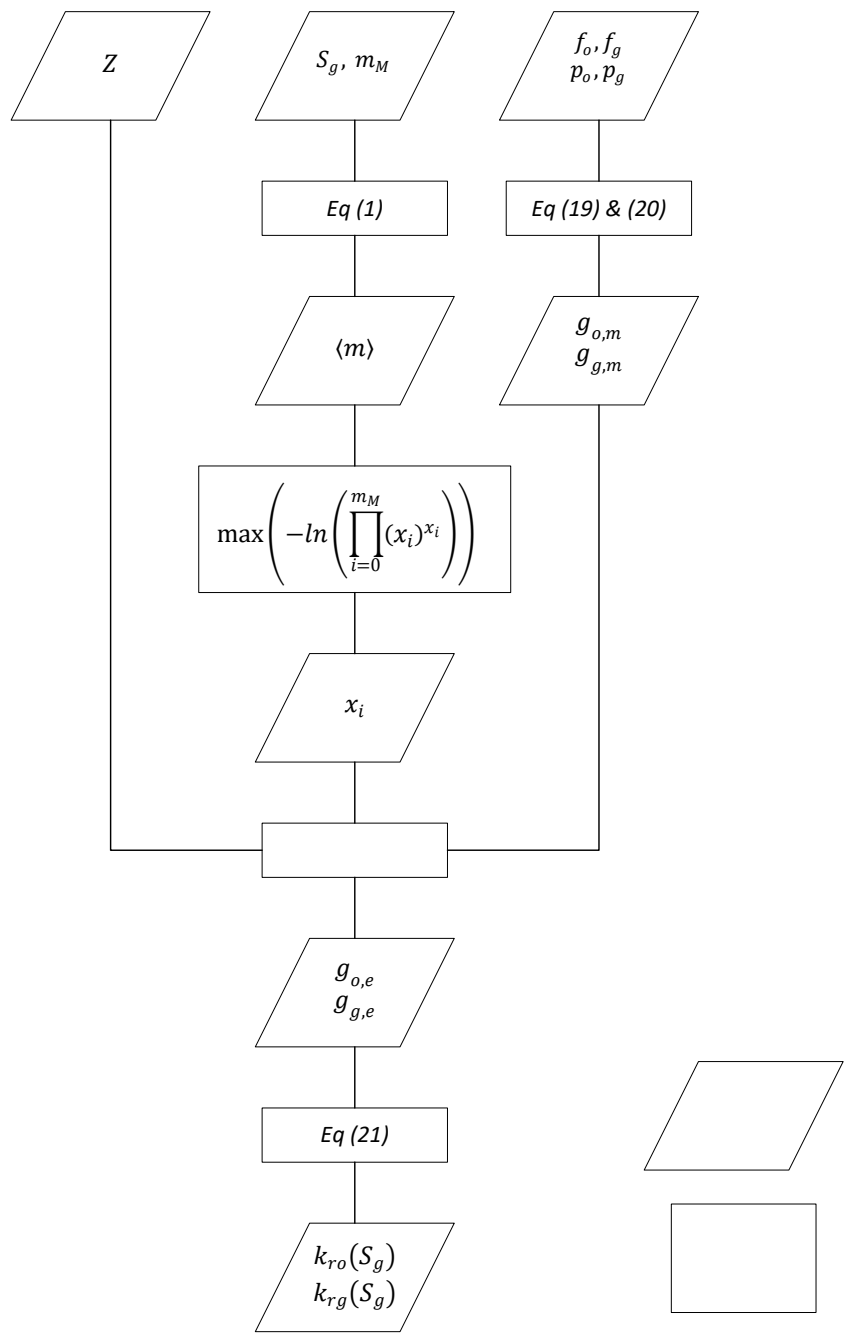

Fig. 1 Flowchart illustrating the process to compute relative permeabilities for oil and gas phases

It should be remarked that the permeabilities for gas and oil determined by formulae (19) to (21) are, in a way, symmetric with regard to each other. Although they were described by introducing the gas bubbles in the oil-filled pores, starting from the condensate droplets in the gas capillaries would result in the same final expressions. In the following, we are mainly concentrating on the analysis of the gas relative permeability, since the oil relative permeability behaves similarly.

In the works Bagudu, McDougall, \& Mackay (2018), Firoozabadi \& Kashchiev (1996), Firoozabadl, Ottesen, \& Mikkelsen (1992), Kashchiev \& Firoozabadi(1993), Li \& Yortsos (1995a), Ioannis N. Tsimpanogiannis \& Yortsos(2002, 2004) and Yortsos \& Parlar(1989), the following qualitative picture of the gas bubble formation and flow was described. A gas bubble is formed in a pore, starting growing from a number of nucleation sites. Until it has filled the whole pore, oil may still flow out, displaced by gas, and withdrawn from the system while the gas remains inside the pore. When the gas bubble fills the whole pore, it may enter the neighboring pores via the pore throats, becoming mobile and, eventually, uniting with the gas bubbles in these pores. In terms of introduced threshold values $f_{o}, f_{g}$ this situation is described as $f_{o}=0$ and $f_{g}=1$, correspondingly. As we will show below, this is not in agreement with the values fitted to the relative permeability data. The reasons for that discrepancy are discussed in Section 4. It will also be shown that the values of $P_{o}$ and $P_{g}$ may be set to unity, and still 
provide a good approximation of the experimental data. They are considered for the future possible extension of the model.

\section{Results and discussion}

\subsection{Effect of the different model parameters}

The gas relative permeability is determined by four parameters: $f_{g}, Z, P_{g}$ and $m_{M}$. The sensitivity to these parameters is essential to determine in order to have an idea about the physical behavior of the model.

We consider a case of high coordination number $Z=24$, as was found for chalk rock characterized by multiple connectivity (Mogensen \& Stenby, 1998; Patsoules \& Cripps, 1983). In the first example, the maximum number of bubbles per capillary will be fixed at $m_{M}=10$, and the shape-factor $P_{g}$ to be 0.25 . The gas bubble threshold $f_{g}$ will vary, assuming values 0.2 , 0.4 and 0.8 . For each value of $f_{g}$ the relative permeability is calculated as a function of gas saturation, as illustrated in Fig. 2a. It can be seen that the effect of the fractional gas threshold is significant. Any change in the threshold will change the critical saturation and the shape of the dependence. A smaller value of $f_{g}$ corresponds to a critical gas saturation closer to the critical saturation predicted by the theory of effective medium alone.

In the next series of calculations, $f_{g}$ is set to 0.4 , and the sensitivity to the coordination number is investigated. The value of the coordination number, $Z$, can vary significantly, from between 4 and 8 for Berea sandstones (Chatzis, Morrow, \& Lim, 1983) to between 14 and 30 for chalk (Patsoules and Cripps, 1983). The coordination numbers of 6,9 and 24 were thus selected for the present analysis. The results are shown in Fig. 2b. For large values of $Z$, the pores in the formation are more connected. Hence, more pores need to be plugged in order to cut the flow. As a result, higher relative permeabilities and lower critical gas saturation are observed.

For the rest of the study, constant values of $Z$ are considered: $Z=6$ for sandstones and $Z=24$ for carbonates, respectively.

(a) 


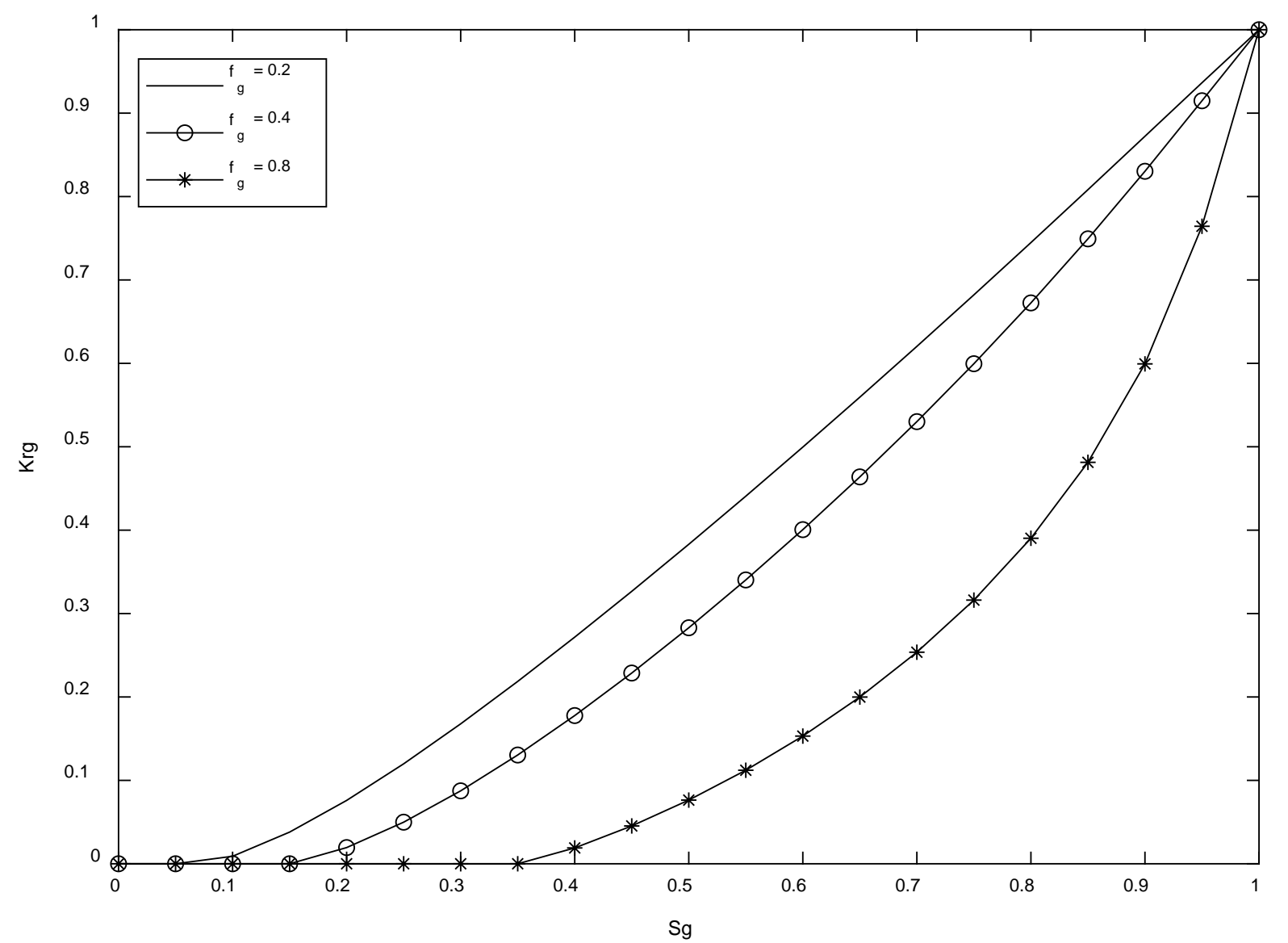

(b) 


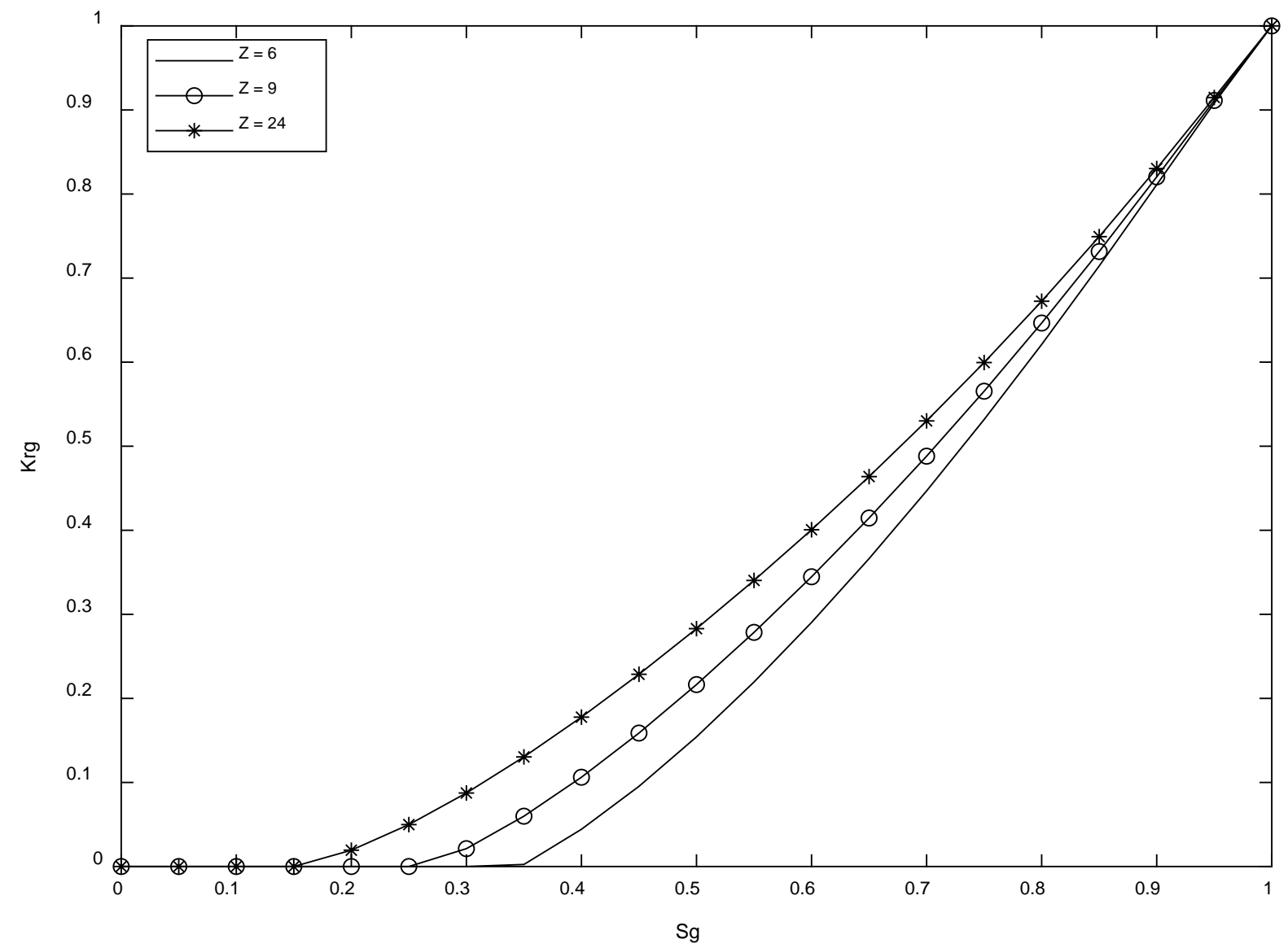

(c) 


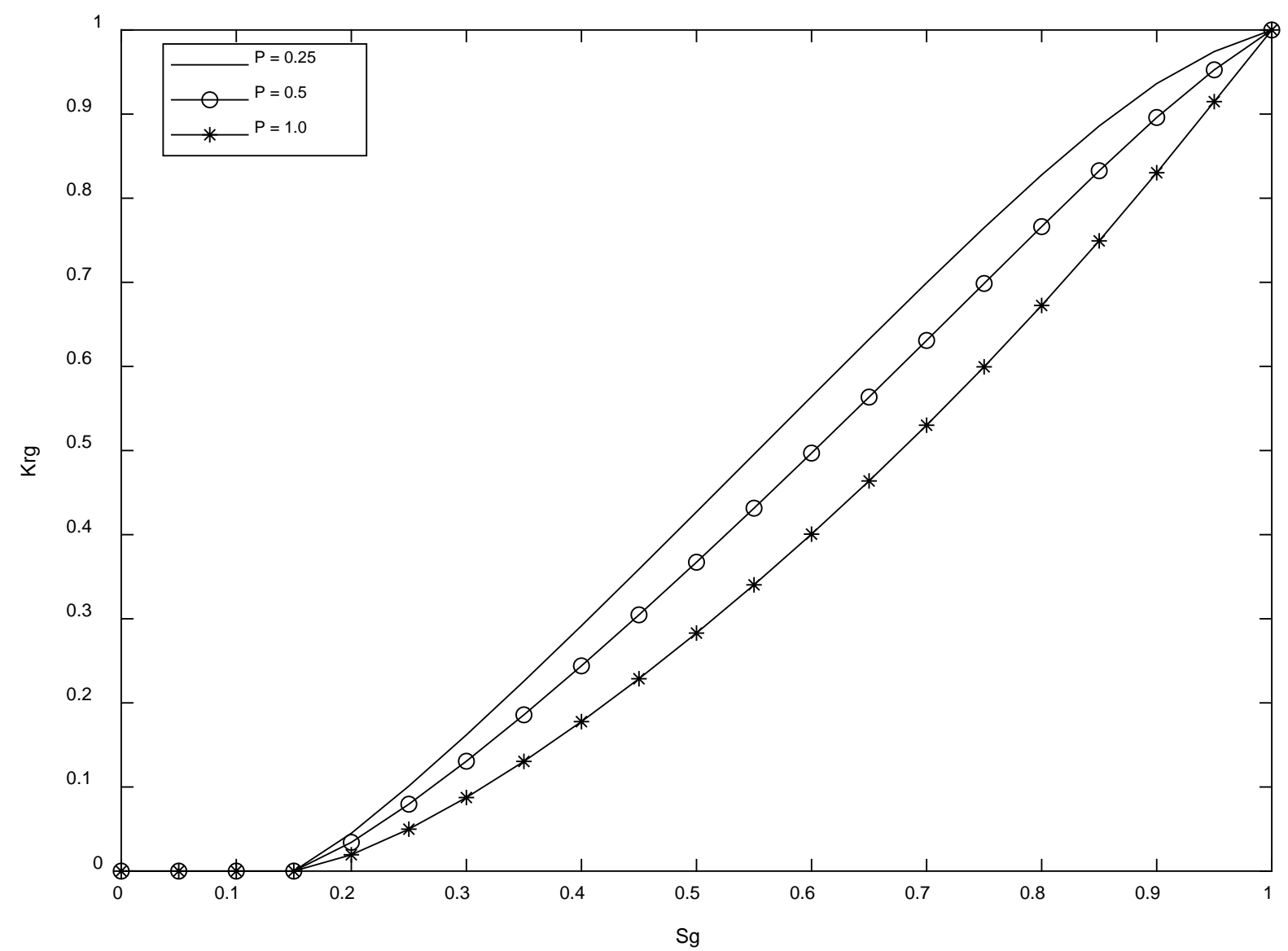

(d) 


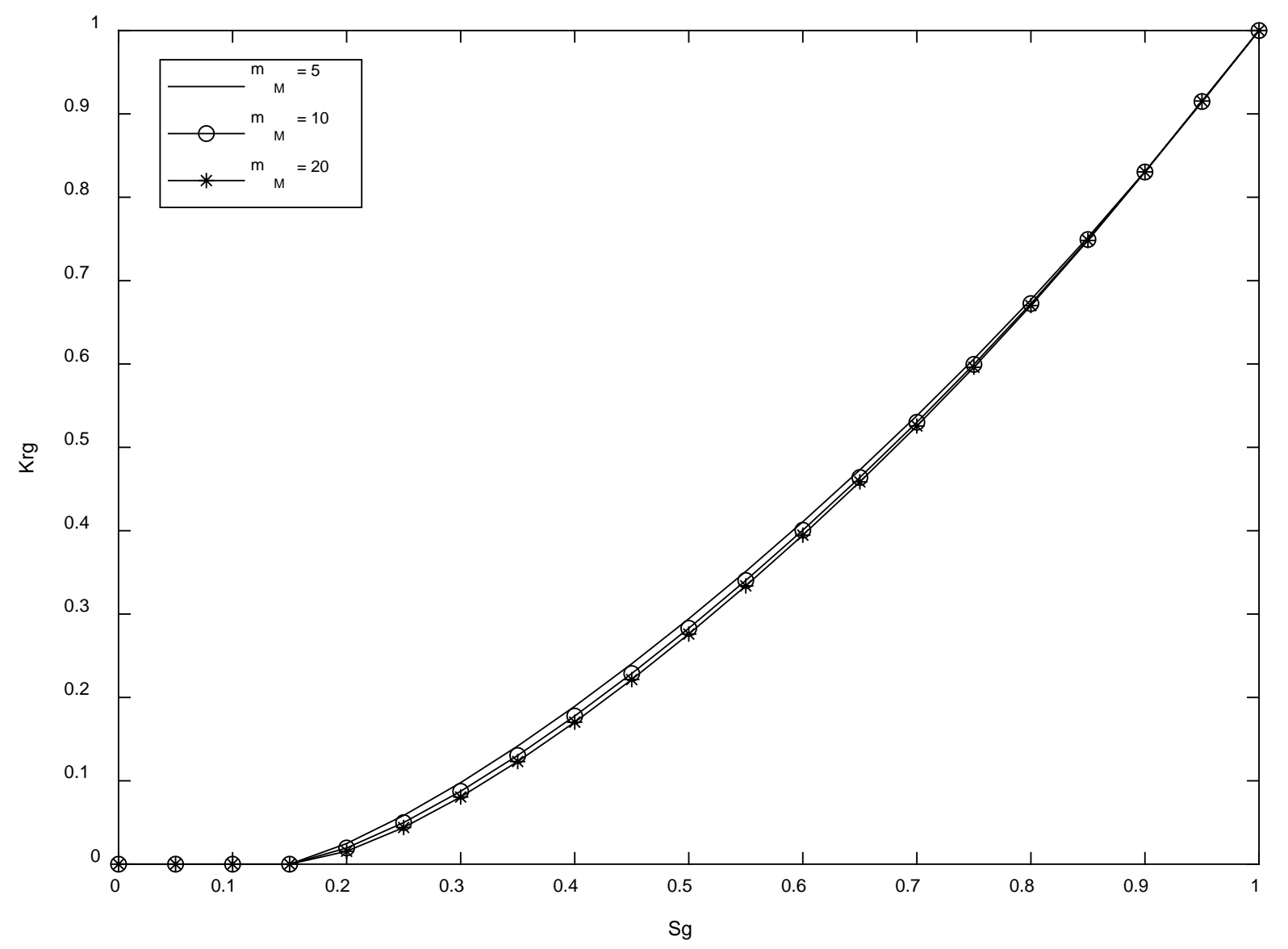

Fig. 2 Sensitivity of the gas relative permeability to the parameters: (a) $f_{g}$; (b) $Z$; (c) $P$, and (d) $m_{M}$,

The effects of parameters $P_{g}$ and $m_{M}$ on the gas relative permeabilities are presented in Fig. 2 c and d, respectively. Both parameters affect only the shape of the dependence. The effect of $P_{g}$ is insignificant, as far as $P_{g}<1$. The effect of $m_{M}$ may probably be neglected. These effects may be connected, to some extent. For example, some calculations (not shown here) indicate that $P_{g}$ may be more important for large values $m_{M}$, corresponding to long capillaries with a small diameter. It should be remarked that $f_{g}$ cannot be selected separately from $m_{M}$, since, according to the model, $f_{g} \cdot m_{M}$ should be an integer. However, this is not a principal limitation, since in practical situations, the values of $f_{g}$ and $m_{M}$ will always express the average values for a given disordered porous medium.

Since the value of $P_{g}$ does not affect the results much; in the rest of the study, it will be fixed to unity.

\subsection{Comparison with the Brooks-Corey model}

In this section, the model is compared with the traditional power law-relative permeability correlations (Corey, 1977). The oil and gas permeabilities may be expressed in the form of

$$
k_{r g}=\left(\frac{S_{g}-S_{g, c r}}{1-S_{g, c r}}\right)^{n_{g}}, k_{r o}=\left(\frac{1-S_{g}-S_{o, c r}}{1-S_{o, c r}}\right)^{n_{o}}
$$

Here $S_{g, c r}, S_{o, c r}$ are critical saturations; and $n_{o}, n_{g}$ are exponents determining shapes of permeabilities. Unlike for the standard Brooks-Corey relative permeabilities, we do not apply 
the values of permeabilities at the residual saturations, like in the standard dependencies, e.g., for oil and water, since our goal is to approximate the permeabilities also in the regions where one of the phases is not flowing.

The Brooks-Corey model is one of the most widely used relative permeability models (Behrenbruch et al., 2018; Corey, 1977). Comparison with our model makes it possible to understand the physical meaning of the parameters constituting the Brooks-Corey model; to extend it onto the gas liberation problems, outside the residual saturation limits; and, hopefully, to become capable of evaluating the Brooks-Corey parameters for a given structure of the porous medium.

The sample plots of the relative permeabilities produced by our model and by the Corey-Brooks formulae are shown in Fig. 3 a and b, for chalk and sandstone, respectively. It can be seen that the agreement between the models is reasonable though not perfect. A characteristic average deviation is within $2 \%$.

(a) 


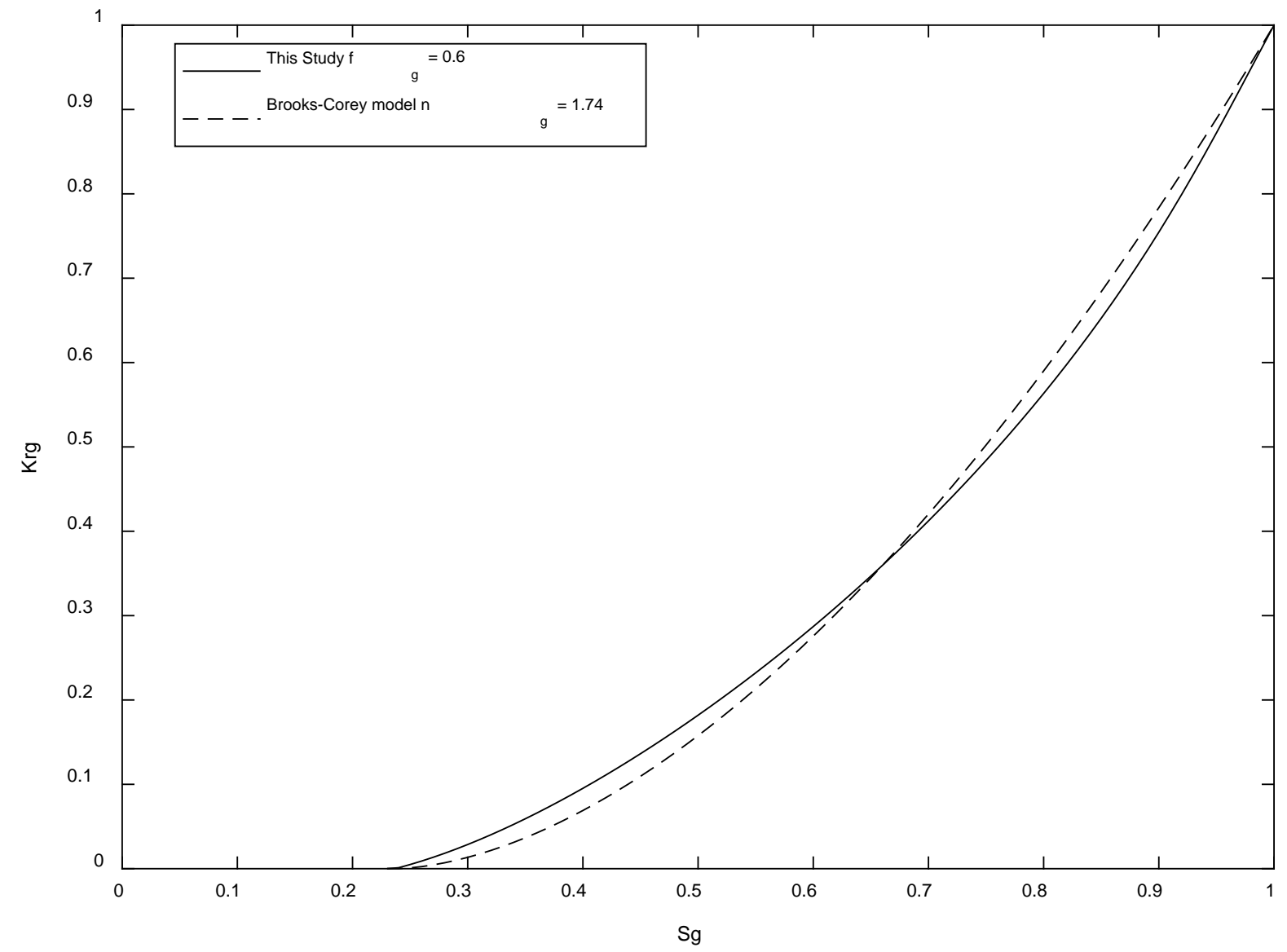

b) 


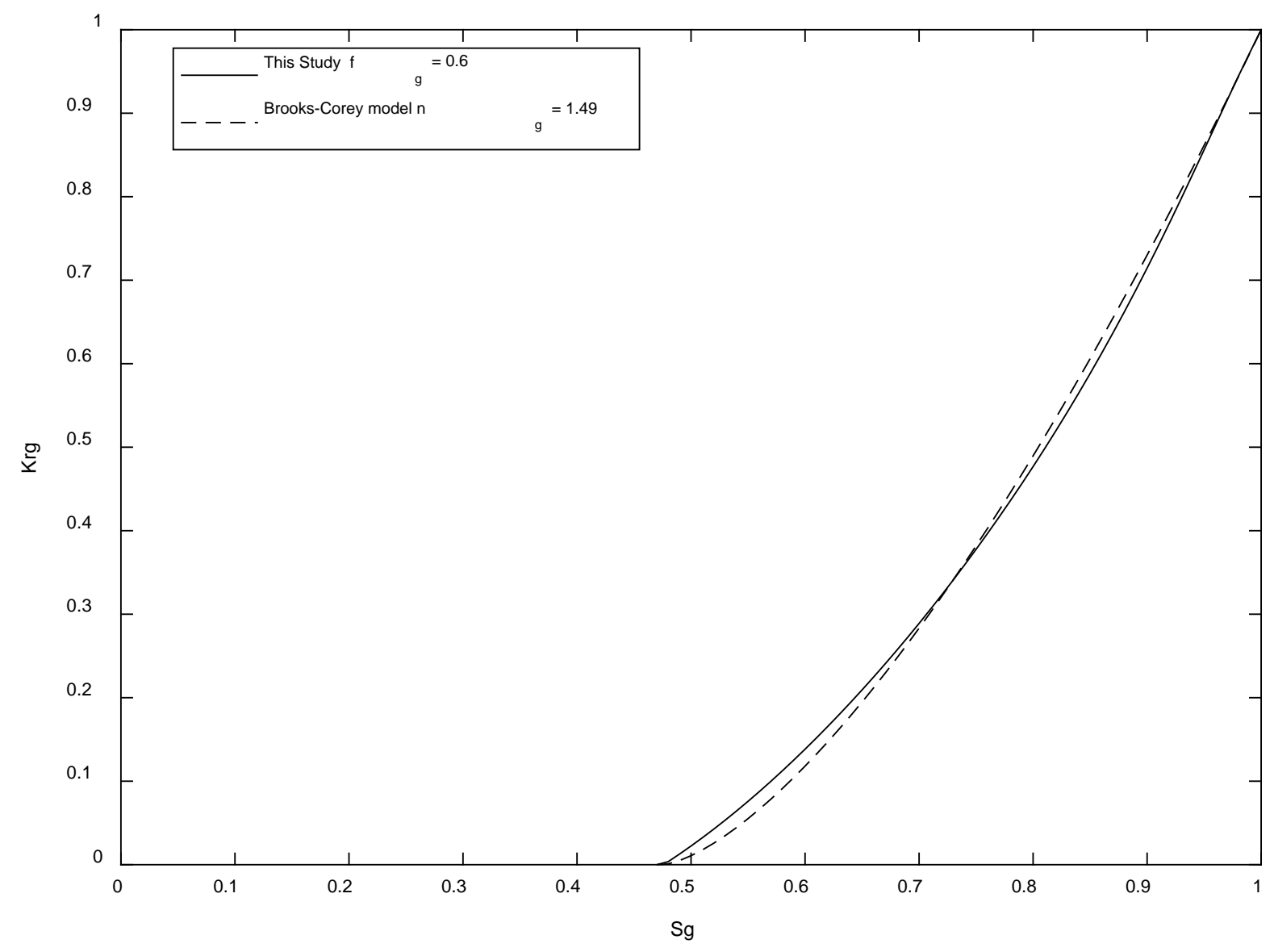

Fig. 3 Comparison between the Brooks-Corey model and this work. (a) chalk rock ( $\mathrm{Z}=24)$; (b) sandstone rock ( $\mathrm{Z}=8)$

The sample computations presented in Fig. 4a shows that the critical gas saturation is linearly dependent on the gas bubble threshold ratio $f_{g}$. For a typical sandstone with the coordination number $Z=6$, the critical gas saturation ranges from 0.15 to 0.55 , while for the typical limestone with the coordination number $Z=24$, the range is from 0.02 to 0.3 . The slope and the intercept of this linear dependence may be represented as universal quadratic functions of $Z^{-1}$ (Fig. 4b and c). A linear dependence would also provide a reasonable approximation, but the quadratic dependence is much more precise.) A combination of these observations makes it possible to relate the value of critical saturation $S_{g, c r}$ approximately, as a function of the model parameters $f_{g}, Z$ :

$$
\begin{gathered}
S_{g, c r} \approx d(Z) f_{g}+b(Z) ; \\
d(Z)=\delta_{0}+\frac{\delta_{1}}{Z}+\frac{\delta_{2}}{Z^{2}} ; b(Z)=\beta_{0}+\frac{\beta_{1}}{Z}+\frac{\beta_{2}}{Z^{2}} ; \\
\delta_{0}=0.27 ; \delta_{1}=4.42 ; \delta_{2}=-10.72 ; \beta_{0}=-2.1110^{-2} ; \beta_{1}=5.3910^{-2} ; \beta_{2}=2.70 ;
\end{gathered}
$$


a)

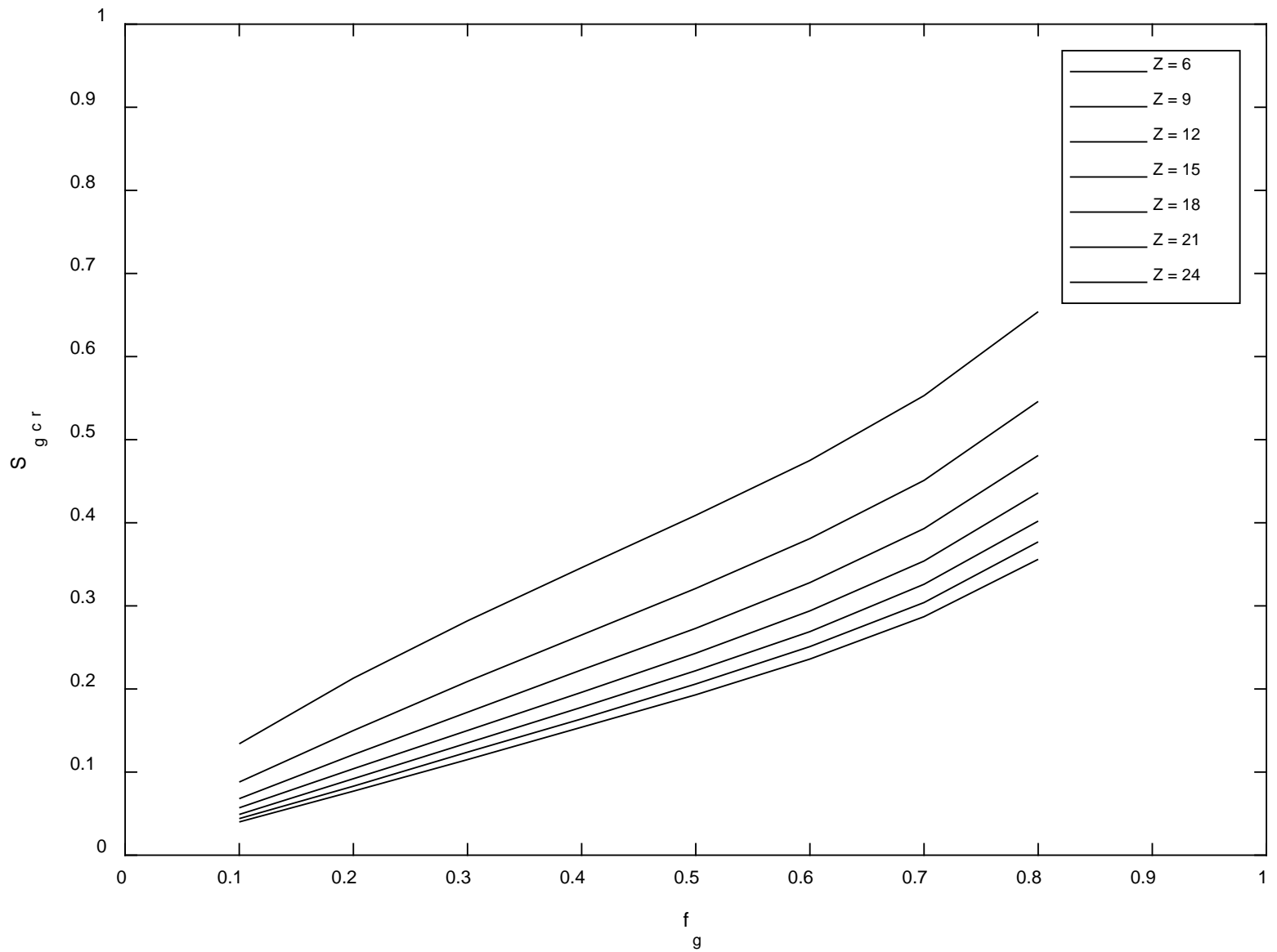

b) 


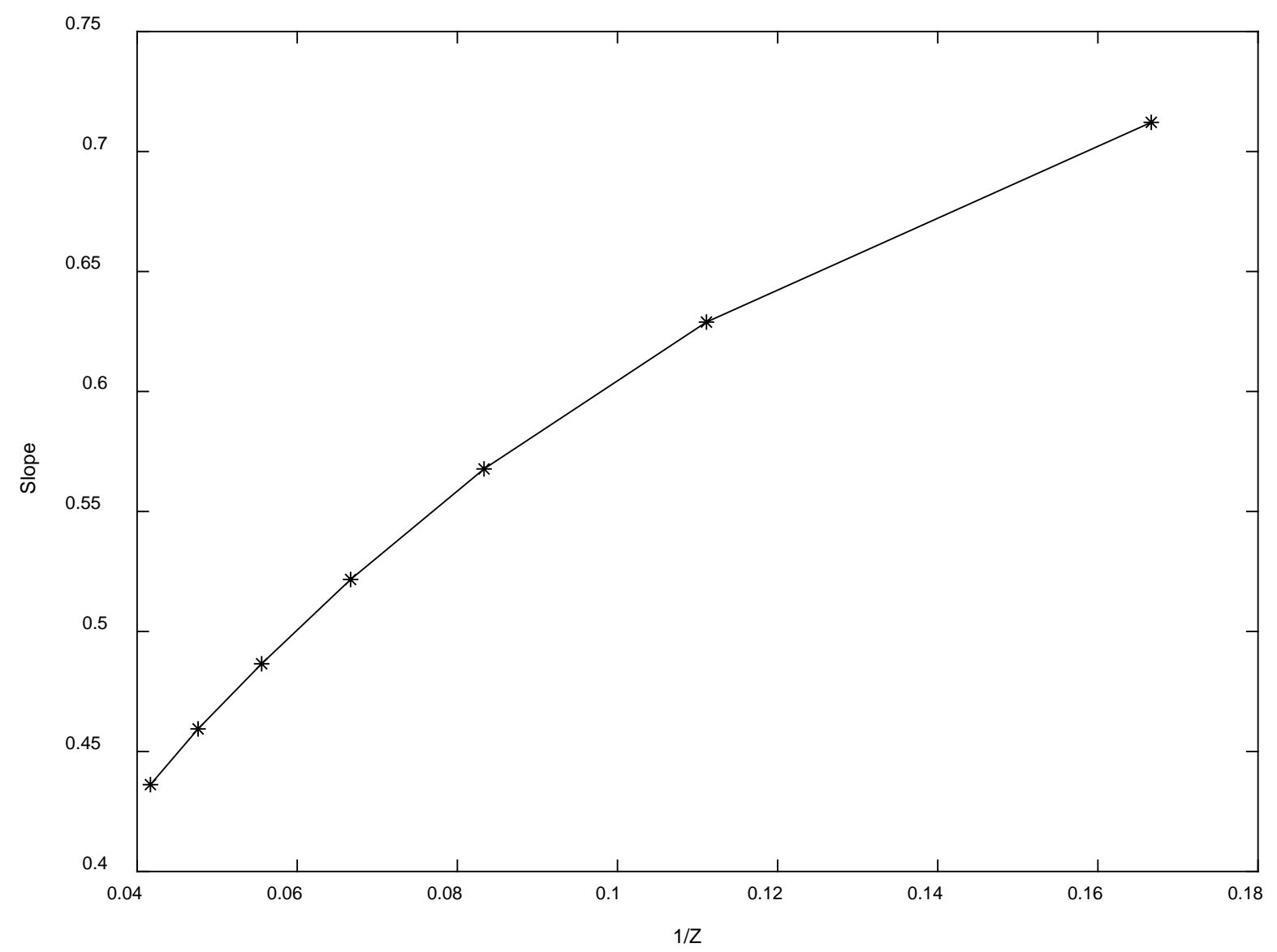

c)

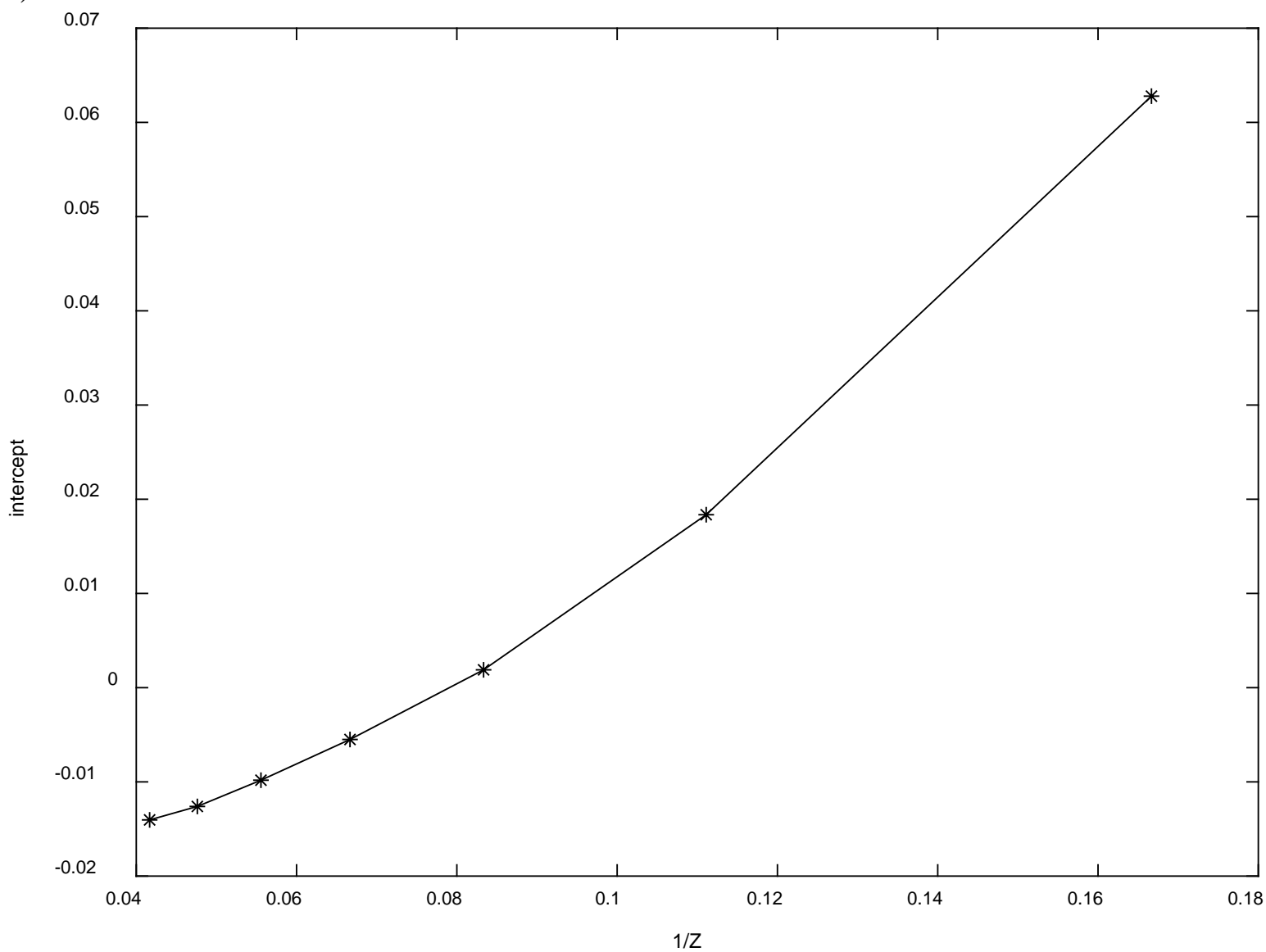


Fig. 4 Critical gas saturation $S_{g, c r}$ as a function of the coordination number $Z$ and the gas bubble threshold ratio $f_{g}$. a) Dependence of $S_{g, c r}$ on $f_{g}$ for different values of $\left.\left.Z ; b\right), c\right)$ Dependences on $Z$ of the slope and intercept for the linear approximation of the dependences plotted in a).

The value of $n_{g}$ may also be expressed as a universal function of $Z$ and $f_{g}$. Under constant $f_{g}$, the dependences of $n_{g}$ on $Z^{-1}$ are approximately linear, within the region of interest (Fig. 5a):

$$
n_{g} \approx l\left(f_{g}\right)+\frac{t\left(f_{g}\right)}{Z}
$$

The dependences $l\left(f_{g}\right)$ and $t\left(f_{g}\right)$ are shown in Fig. 5 b and c. Within a reasonable accuracy, they are fitted by the quadratic functions:

$$
\begin{gathered}
l\left(f_{g}\right)=\lambda_{0}+\lambda_{1} f_{g}+\lambda_{2} f_{g}^{2} ; t\left(f_{g}\right)=\tau_{0}+\tau_{1} f_{g}+\tau_{2} f_{g}^{2} ; \\
\lambda_{0}=1.19 ; \lambda_{1}=-0.35 ; \lambda_{2}=2.45 ; \tau_{0}=0.27 ; \tau_{1}=-0.46 ; \tau_{2}=-6.07
\end{gathered}
$$

a)

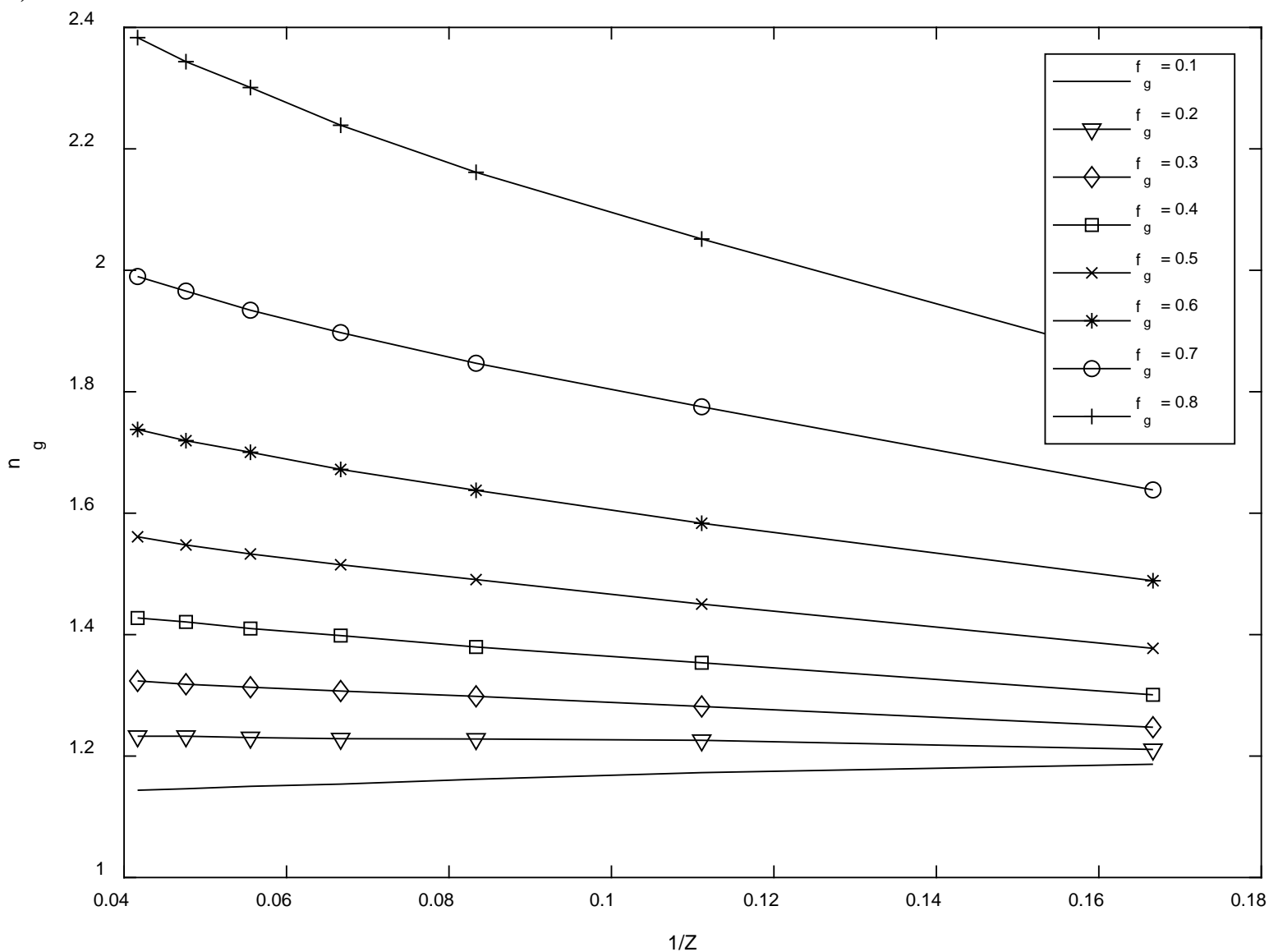

b) 


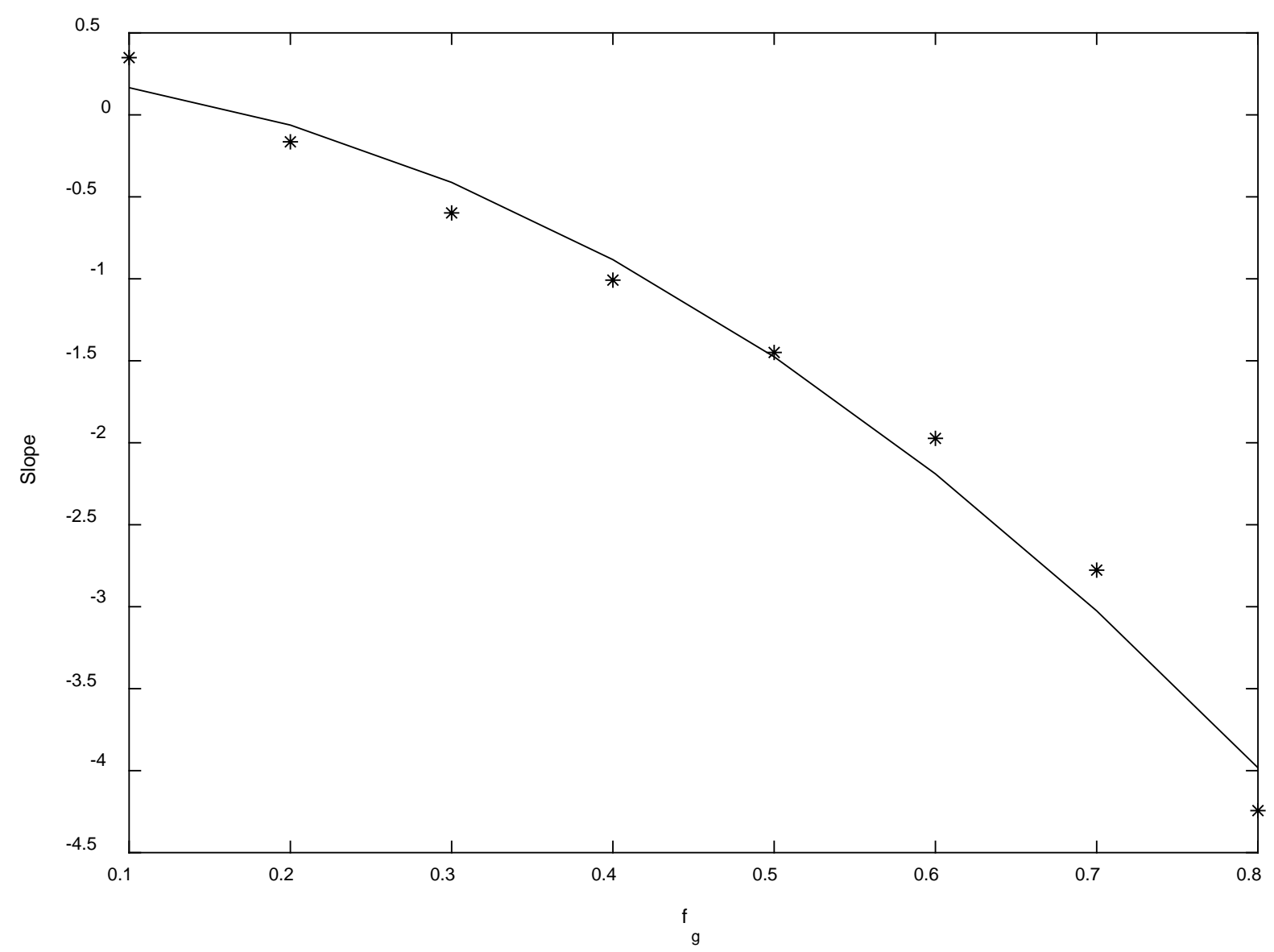

c)

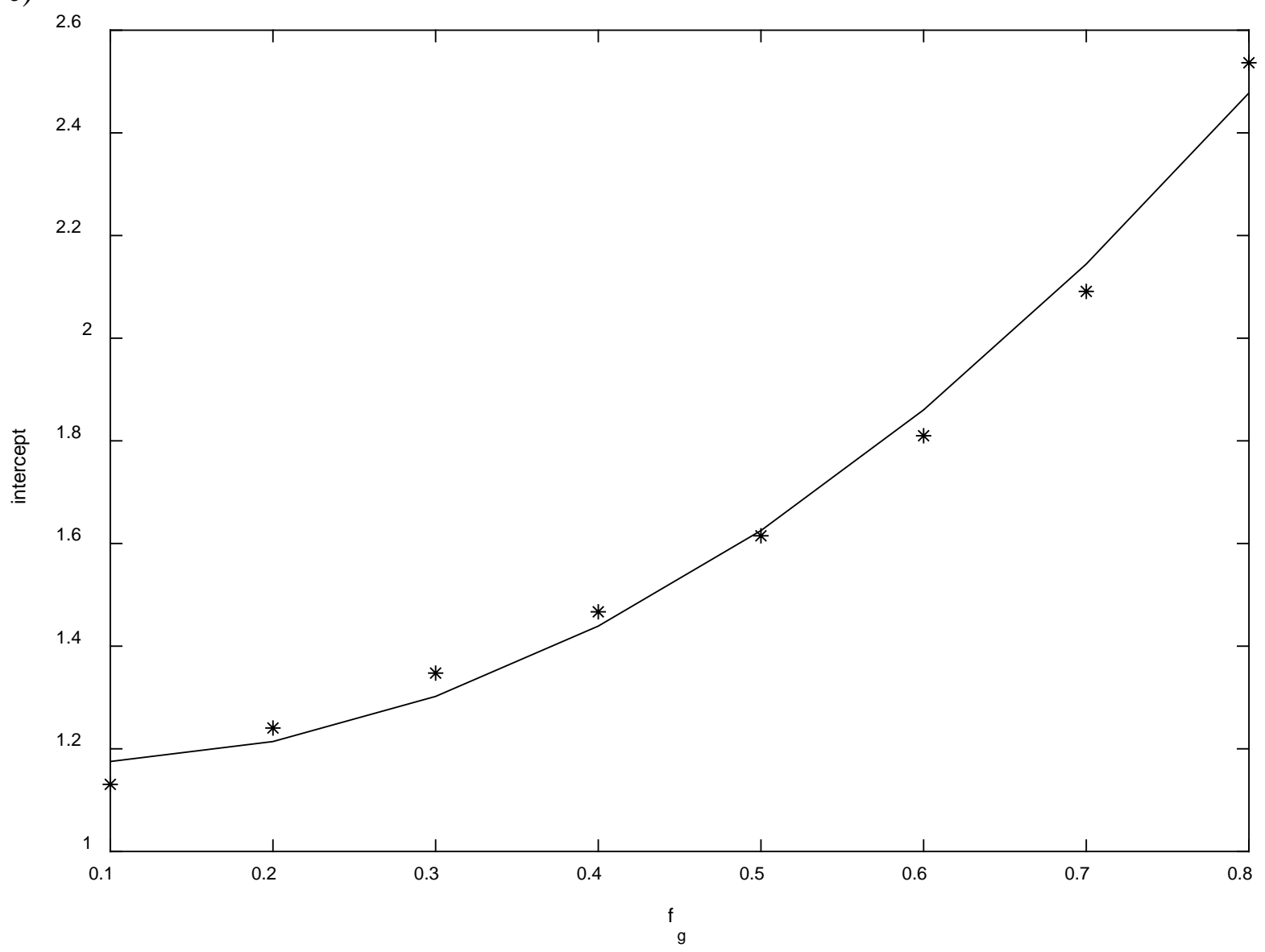


Fig. 5 The Brooks-Corey exponent $n_{g}$ as a function of the coordination number $Z$ and the gas bubble threshold ratio $f_{g}$. a) Dependence of $n_{g}$ on $Z$ for different values of $\left.\left.n_{g} ; b\right), c\right)$ Dependences on $Z$ of the slope and intercept for the linear approximation of the dependences plotted in a).

In this way, the Corey-Brooks approximations for the relative permeabilities may be determined if the geometry of the porous medium (the value of $Z$ ) and the physics of condensation $\left(f_{g}\right)$ are known.

\subsection{Comparison with experimental data}

In this study, three data sets from the literature have been chosen for the model verification: The data on a carbonate rock by Afidick, Kaczorowski, and Bette (1994) and by Gravier et al. (1986); and the data for a sandstone reservoir rock by Chen, Wilson, and Monger-McClure (1999).

Gravier et al. (1986) studied the effect of precipitation of the condensate droplets from gas condensate on the relative permeability for gas. The experiment was conducted on eight samples from a Middle Eastern retrograde condensate gas carbonate field using a ternary pseudoreservoir fluid of methane/pentane/nonane. Afidick, Kaczorowski, and Bette (1994) studied the performance loss of the Arun gas condensate limestone field due to the droplet condensation using the well-test data. Chen, Wilson, and Monger-McClure (1999) measured the effect of condensation on the effective permeability of the two North Sea reservoirs with the properties characteristic of the sandstone reservoirs experimentally.

Assume that the irreducible water saturations are constant for all the data points from a single experiment. The pores filled with immobile water will not contribute to the relative permeability to any of the phases if it is normalized to the permeability at the irreducible water saturation. Thus, the data may be normalized in such a way that $S_{c}+S_{g}=1$ and $k_{r g}^{N}=$ $k_{r g} / k_{r g}$ max

After normalizing the data, the model was fitted using $Z=24$ for the carbonate reservoirs and 8 for the sandstone. The value of $f_{g}$ was the only fitting parameter. The value of $P_{g}$ was set to be unity. Thus our model was able to fit the experimental data with fewer parameters than the Brooks-Corey model; this would be equivalent to obtaining the exponents in the Brooks-Corey model out of the values of the critical saturation. The results are presented in Fig. 6-8.

It can be seen that the model fits experimental data with reasonable accuracy. In all of the cases, the value of $f_{g}$ lies between 0.2 and 0.5 . Moreover, the same value of $f_{g}$ may be used to give the best fit for all the cores from a specific study (e.g., the value of 0.4 for the study of Gravier et al. (1986)). This is seen from the last plot in Fig. 6i, where all the data are compared with a single model plot. This comparison indicates that our model really reflects the physical mechanisms behind the formation of the relative permeability for a given geometry of the porous space. For the cores of the same origin, with similar pore space geometries, the relative permeabilities may be obtained with the same parameters for our model. This is valid for both chalk and sandstone rocks.

The value of coordination number equal to 24 is related to the specific pore structure of carbonates, as previously reported in the literature (Patsuoles and Cripps, 1983; Mogensen and Stenby, 1998). However, this value is unusual for the pore network simulations and capillary micromodels. A question may arise, whether a more traditional value of the coordination 
number may be used for fitting. We have re-fitted the experimental data with the value of $Z=$ 6 (the dashed line in Fig. 6i). The accuracy of fitting did not change much (10.3\% against $10.8 \%)$, most of which may be attributed to scattering of the experimental data. However, such an important parameter as the value of the gas residual saturation was not captured by the new fitted curve. The physics behind the relative permeabilities in chalks is better captured when a more interconnected porous space is assumed.

While most of the experimental works provide only gas relative permeabilities, the work of Afidick, Kaczorowski, and Bette (1994) contains also few measured points for the liquid relative permeability. This has made it possible to produce the liquid relative permeability curve for this data, with the same values of $Z, f_{g}$, and a newly fitted value of $f_{o}=0.6$ (Fig. 7). The fit is satisfactory, but a limited number of reported experimental points does not allow us to make more definite conclusions.

a)

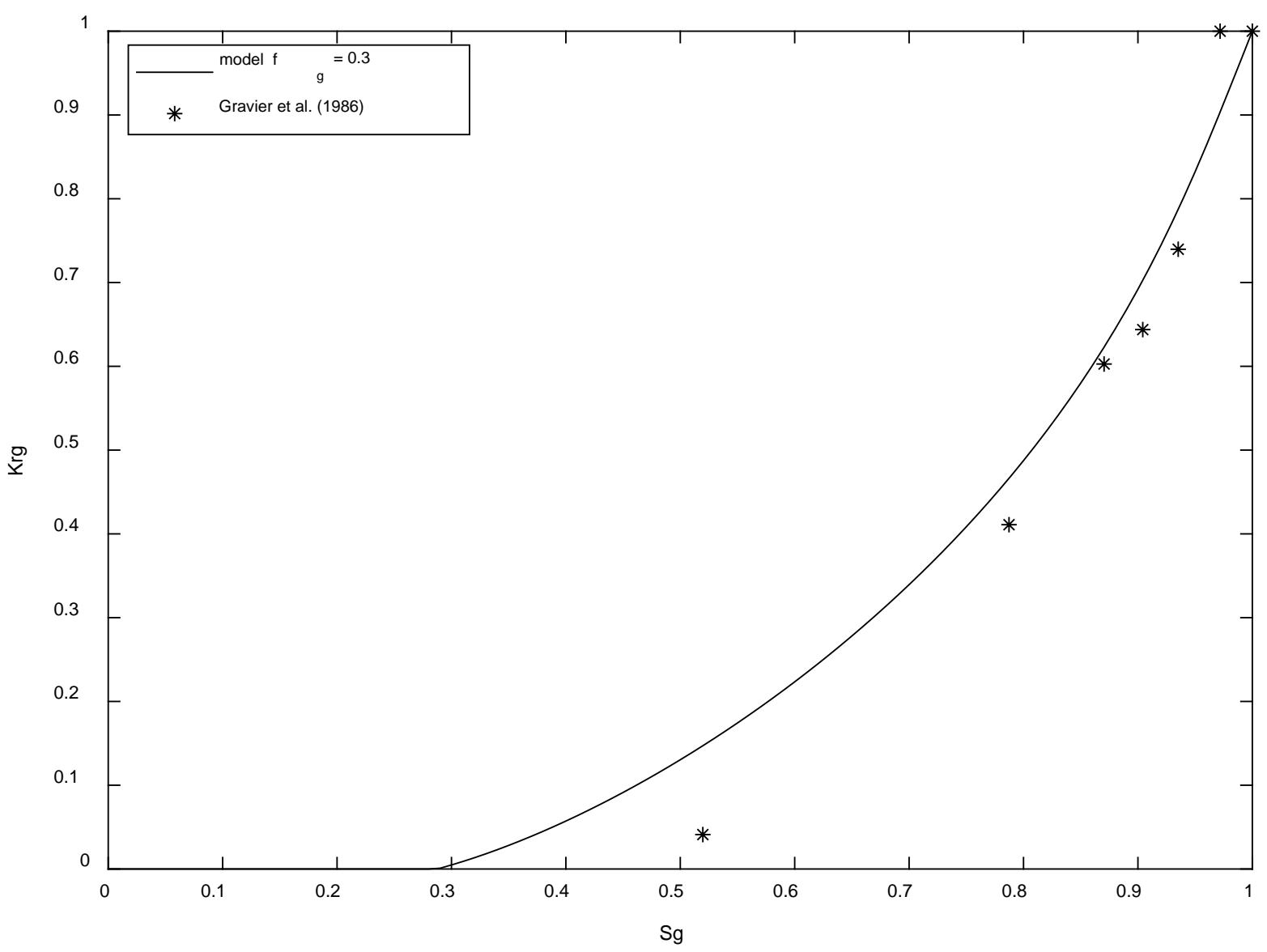


b)

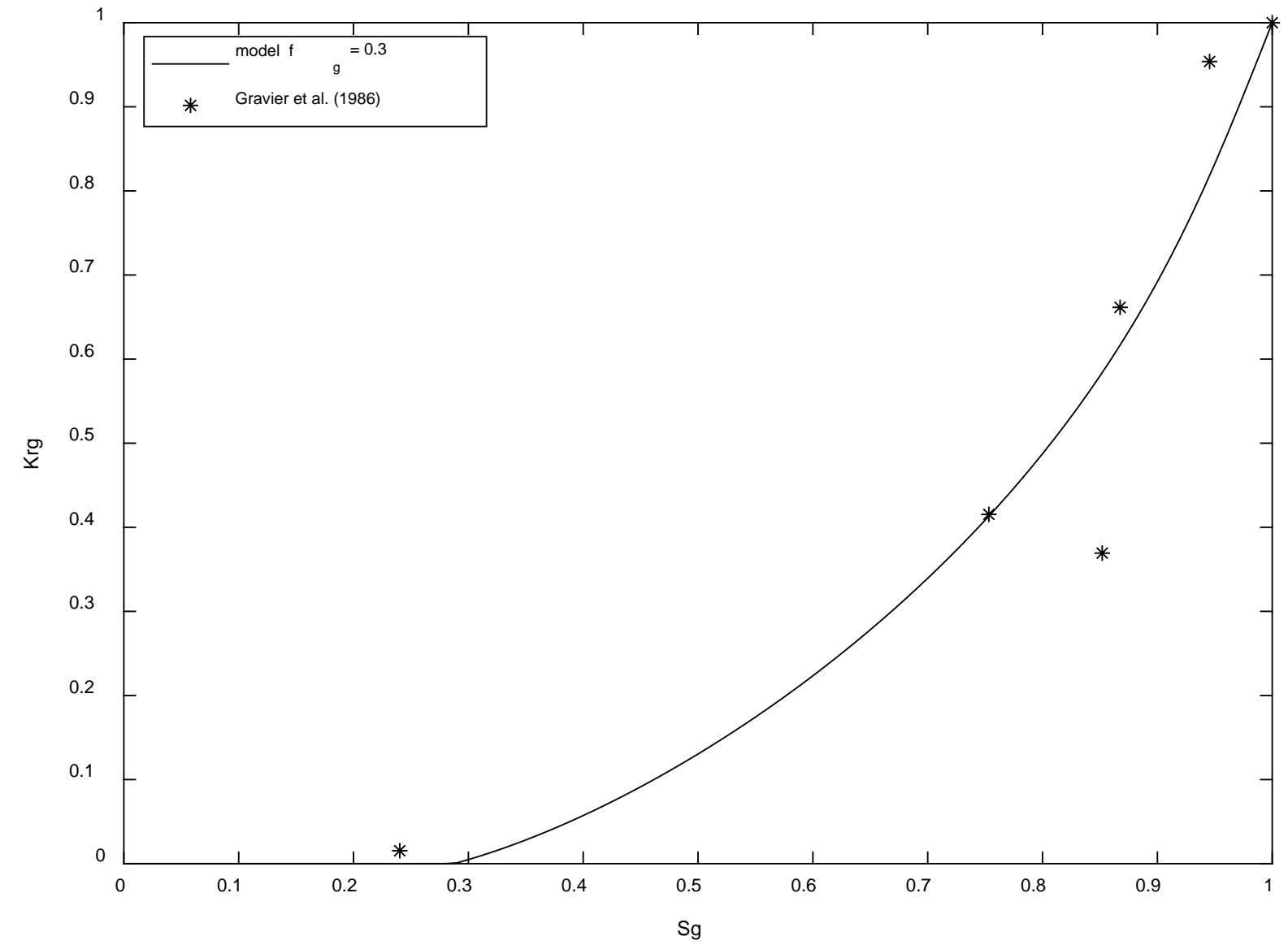

C) 


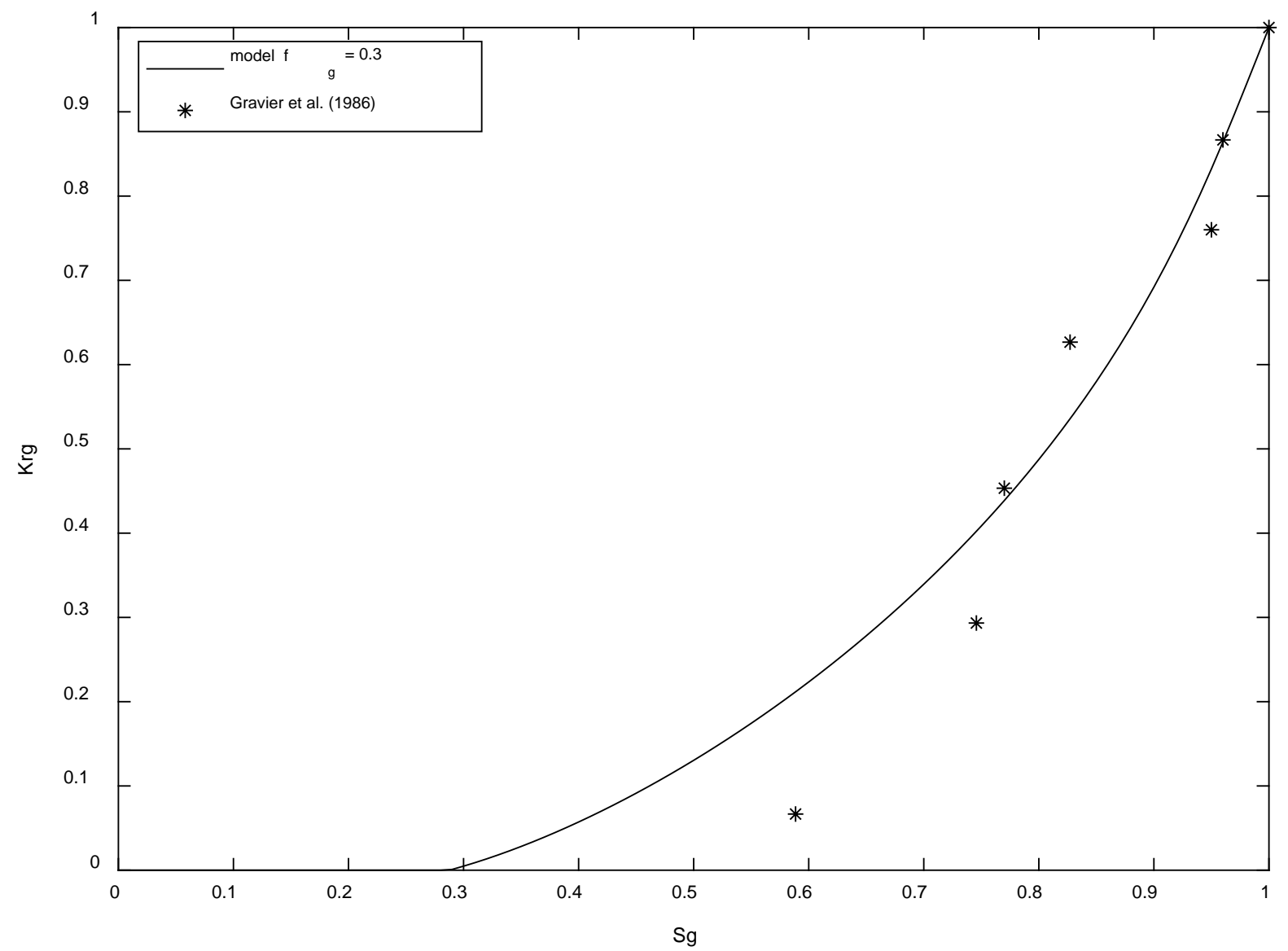

d)

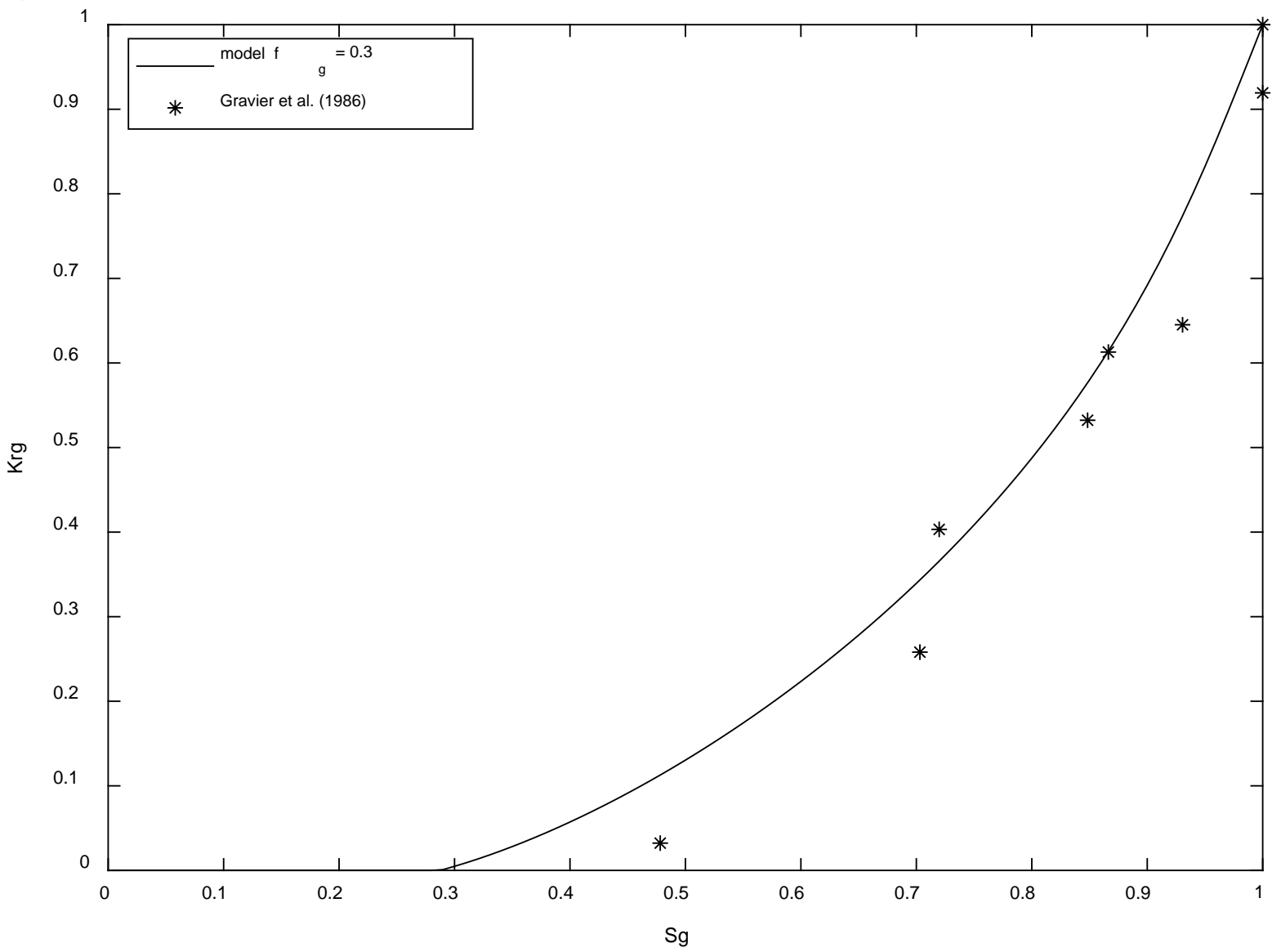


e)

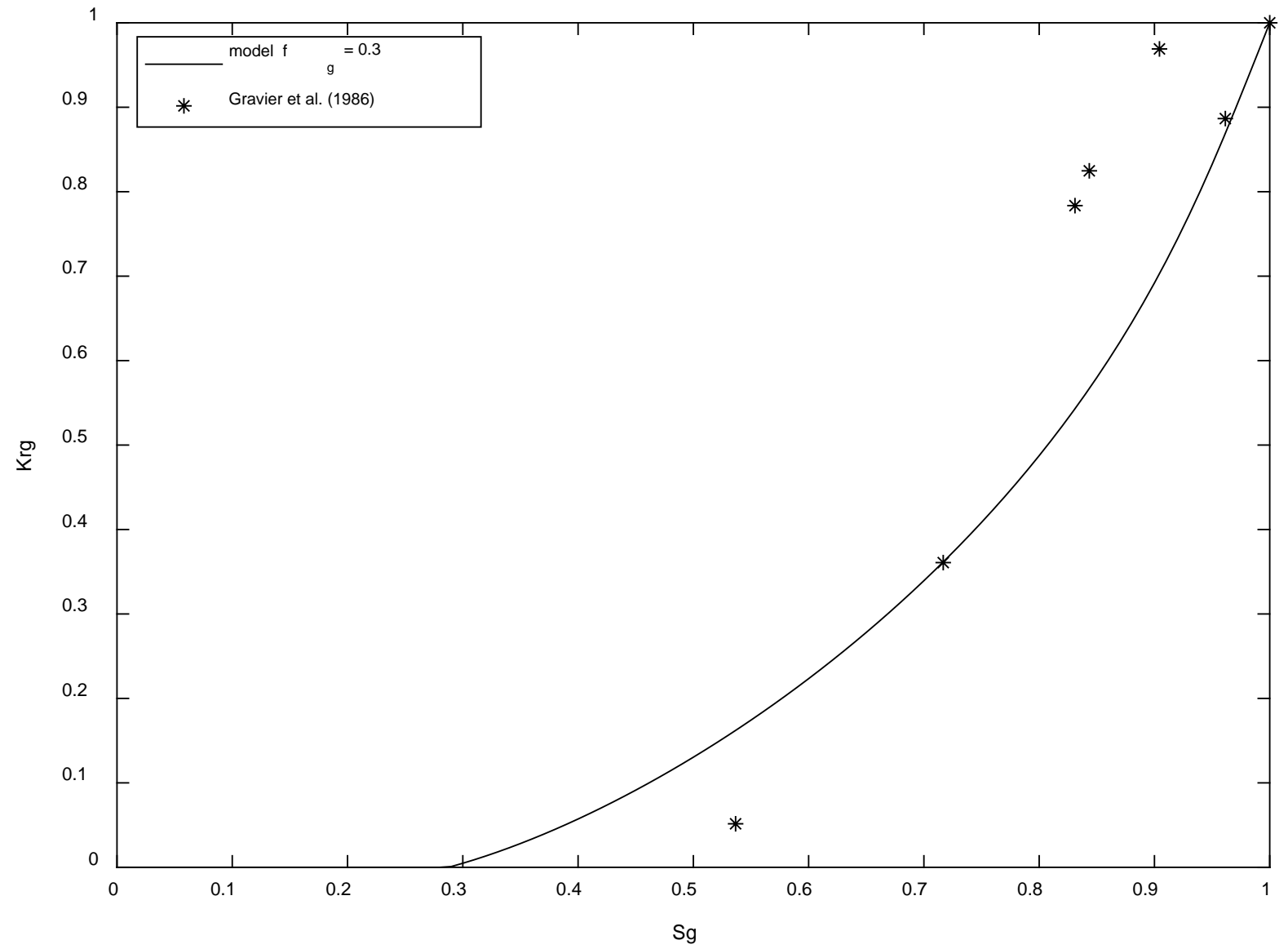

f) 


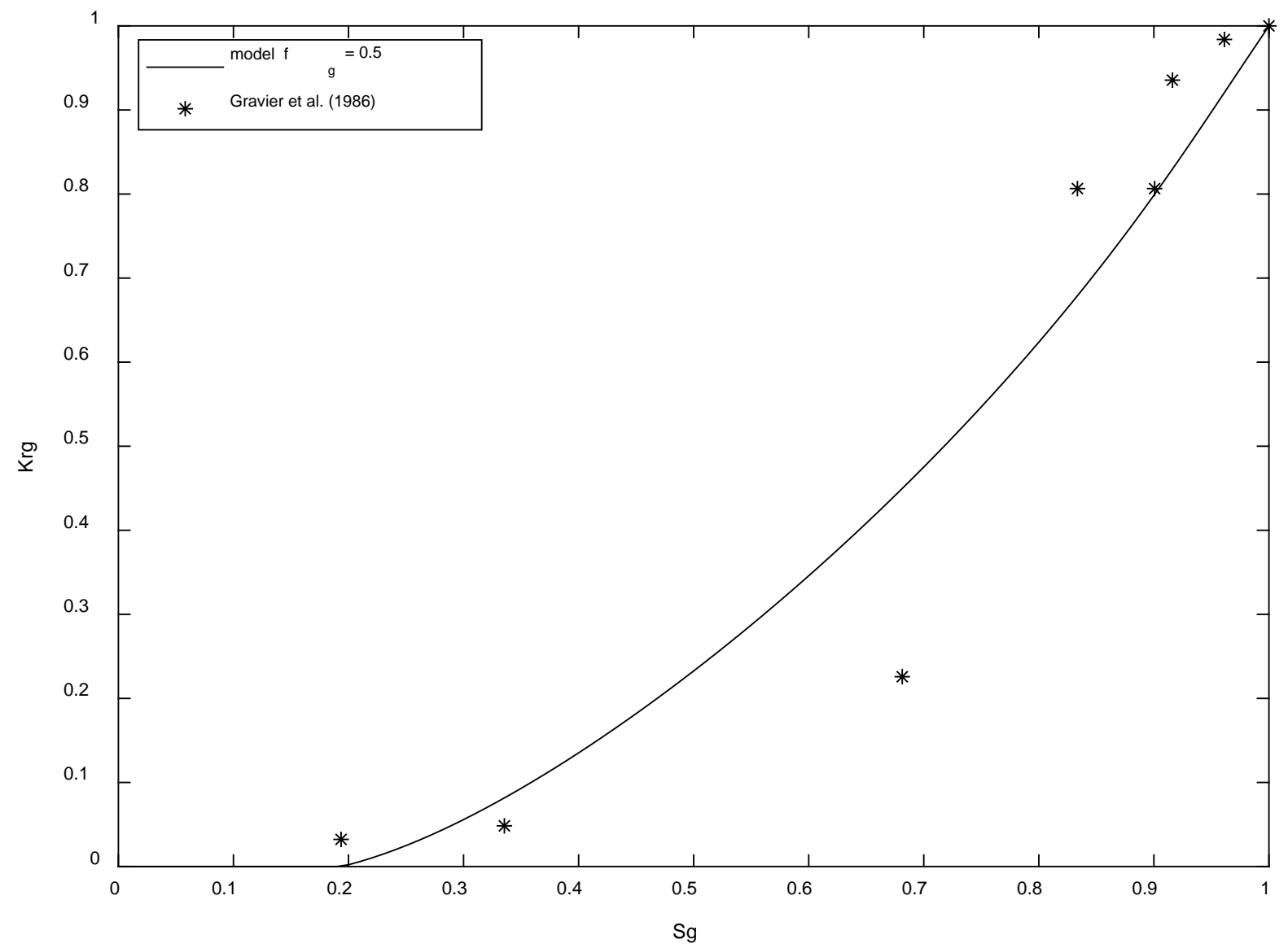

g)

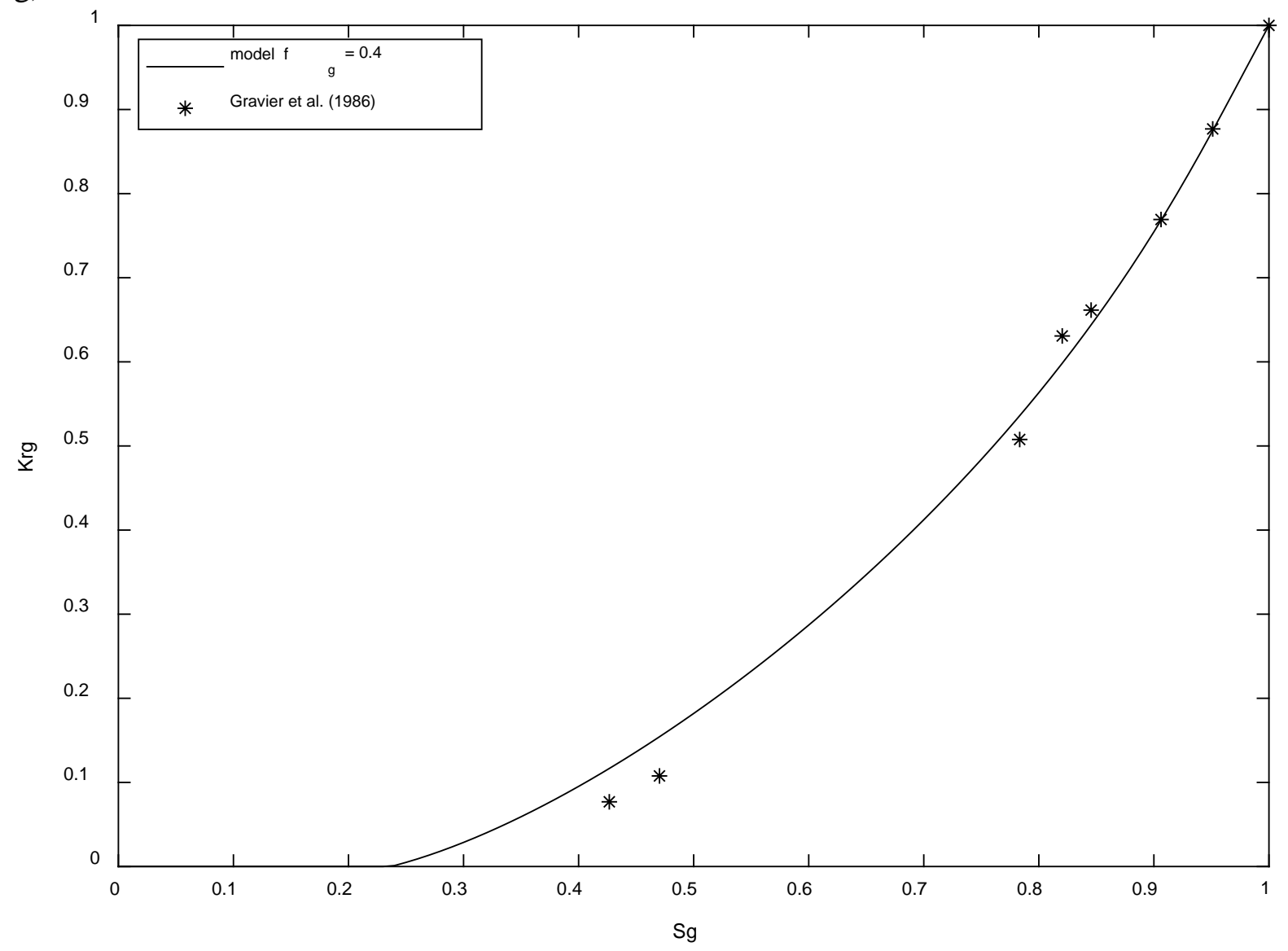


h)

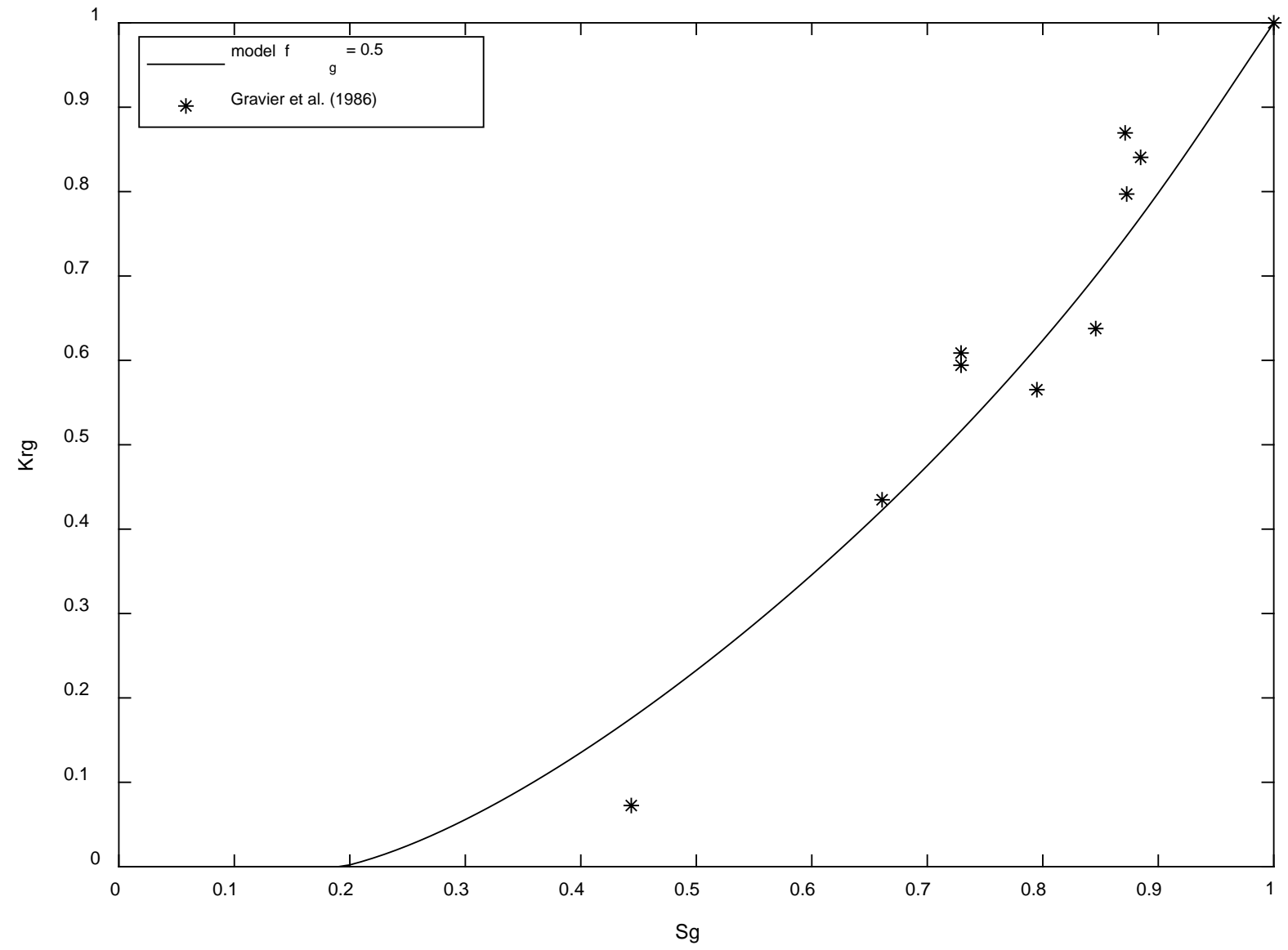


i)

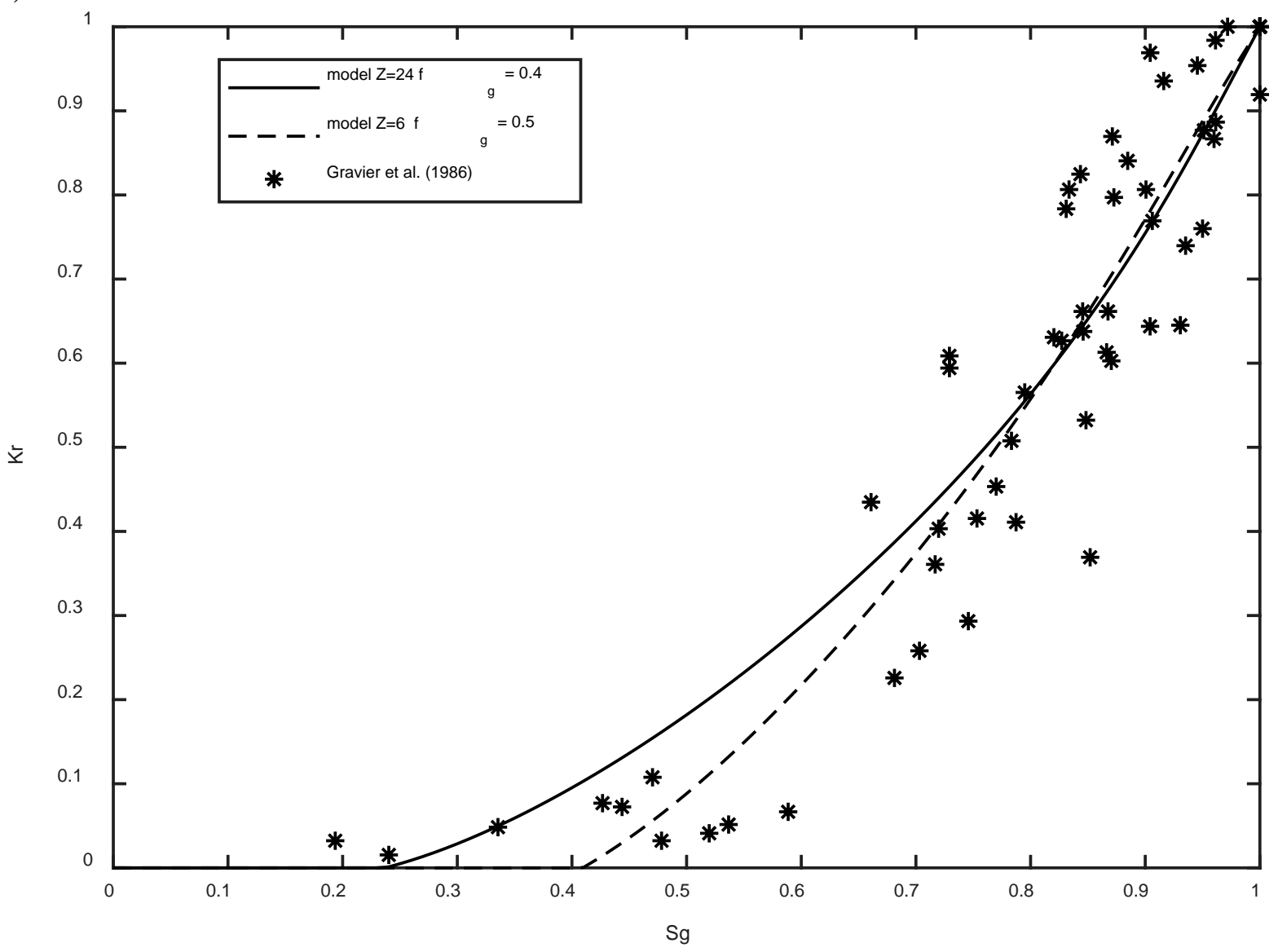

Fig. 6 The model adjusted to the data by Gravier et al. (1986). A carbonate rock $(Z=24)$ Plots (a) to (h) are for the cores 1 to 8 , respectively. The plot (i) presents all the cores together. 


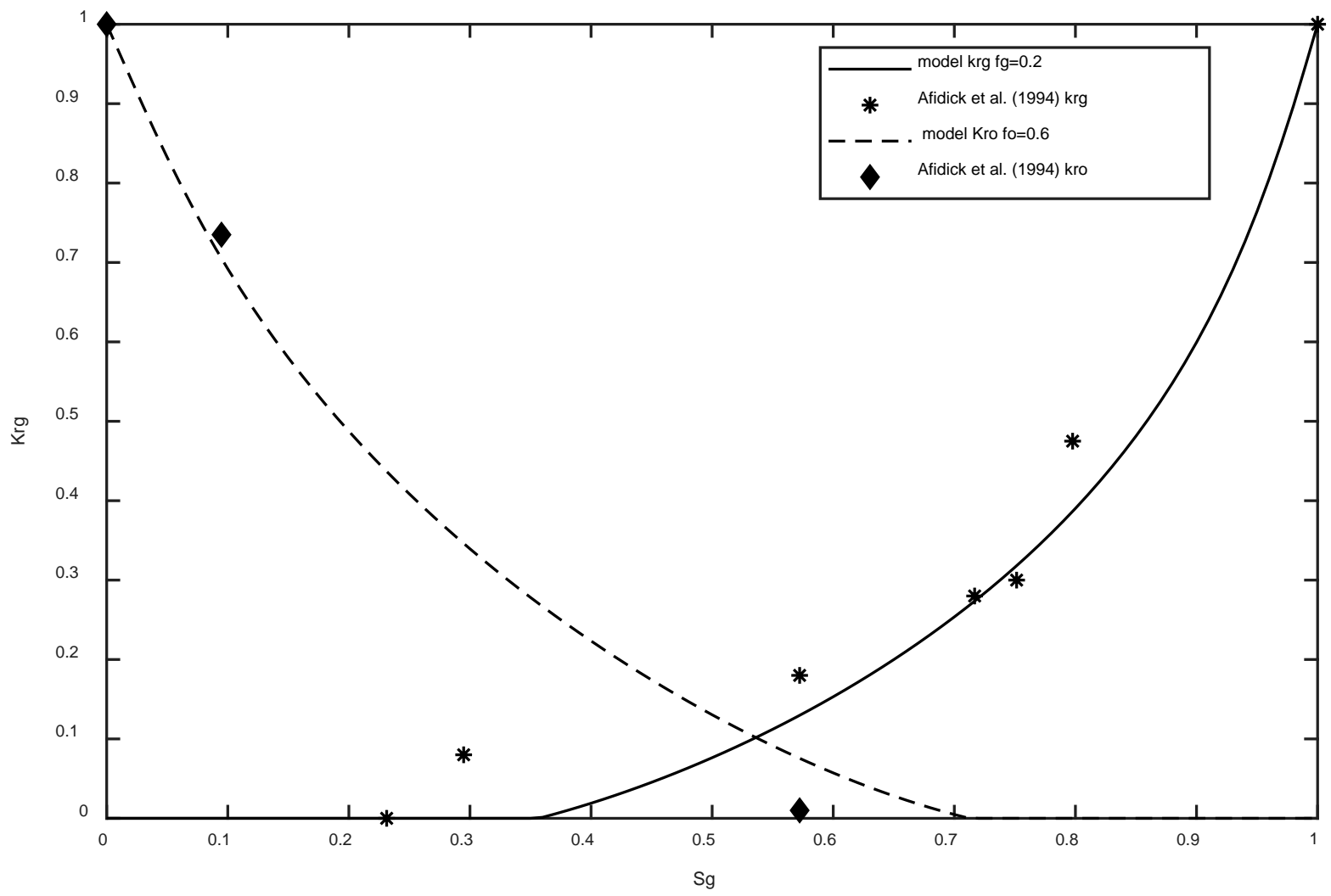

Fig. 7 The model adjusted to the data by Afidick, Kaczorowski, and Bette (1994). Carbonaceous rock $(Z=24)$.

a)

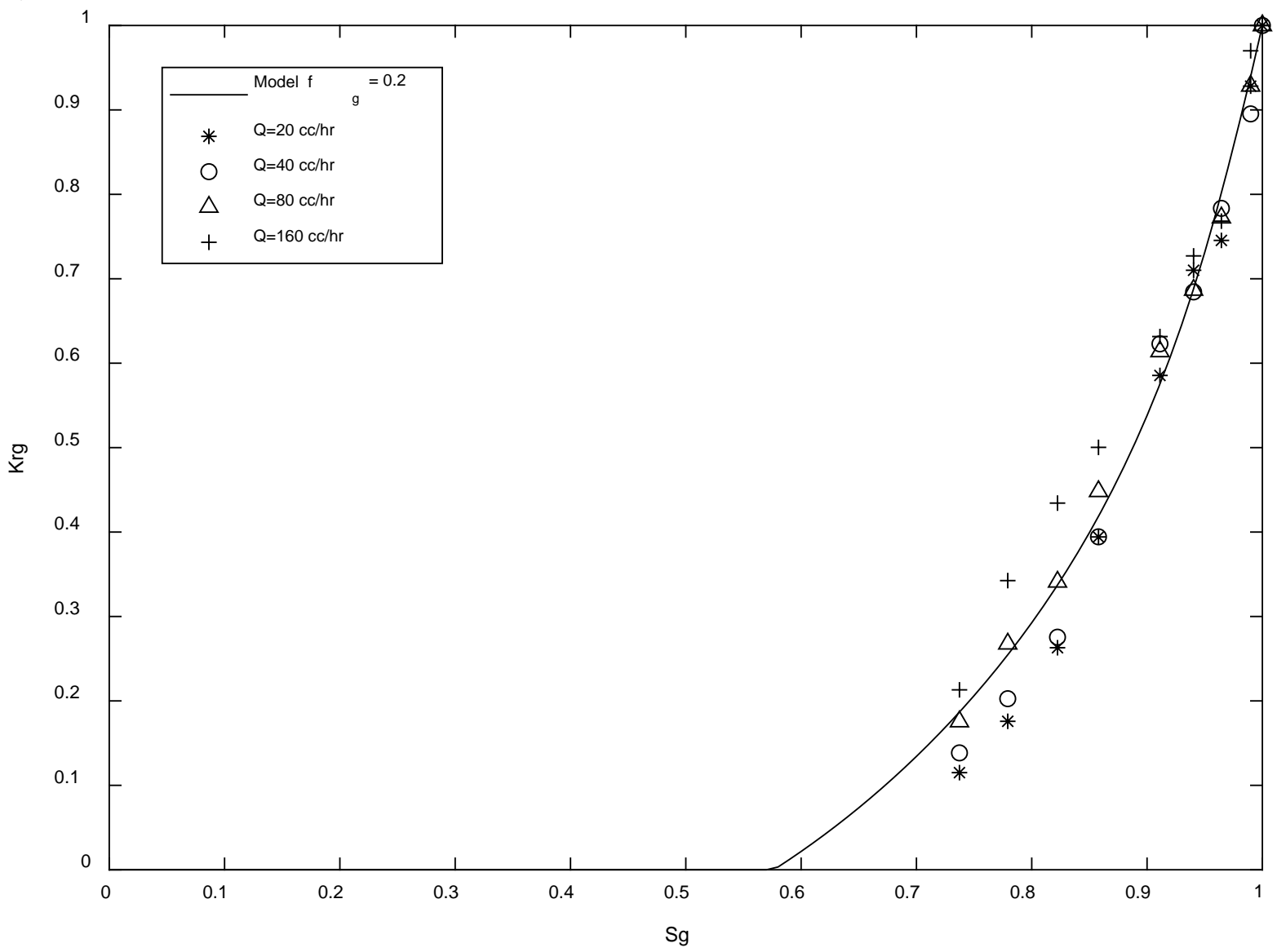


b)

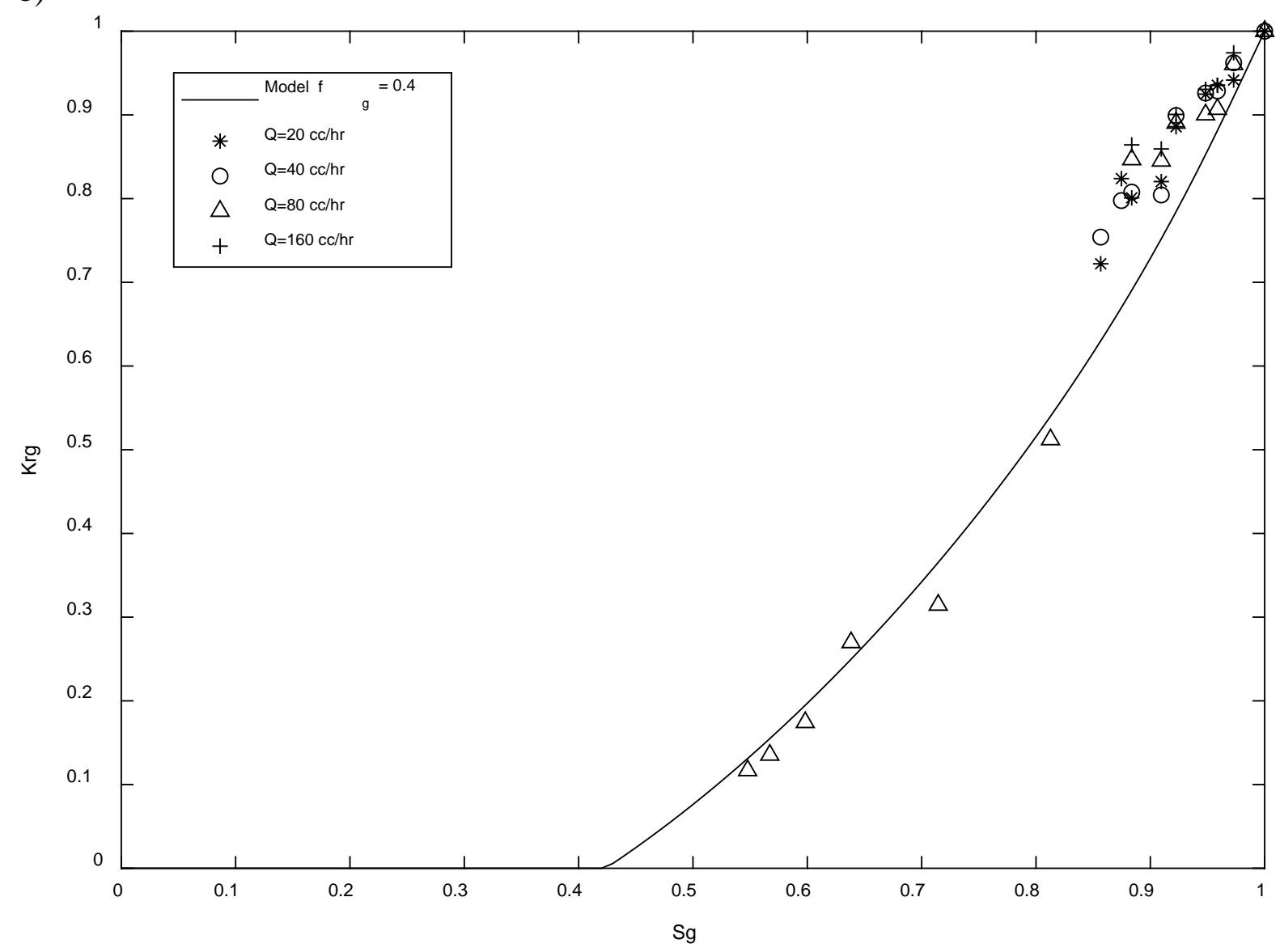

Fig. 8 The model adjusted to data for cores from reservoirs A (a) and B (b), by Chen, Wilson, and Monger-McClure (1999). A sandstone rock $(Z=8)$.

\section{Limitations of the model}

The proposed model has a number of limitations. As shown above, these limitations still allow the model to predict the behavior of the relative permeabilities and to relate them with the microscopic geometrical parameters. This validates the application of the model. However, its limitations still have to be formulated explicitly, in order to be able to spread the model onto more complex cases.

The equal pore sizes, equal numbers of nucleation sites in each pore, and a regular lattice are common assumptions in the percolation models, balancing their simplicity and realism. Some of these assumptions may probably be relaxed. E.g., stochastic pore size distribution Bedrikovetsky (1993) and Selyakov \& Kadet (1996) may be more adequate, although more difficult to implement.

We consider percolation by bonds, not by sites. The volumes of sites have been neglected. According to studies (Kashchiev \& Firoozabadi, 1993; Ioannis N. Tsimpanogiannis \& Yortsos, 2002), nucleation always happens in sites. The subsequent filling of the porous medium involves filling of the sites. Thus, it is an assumption that has to be overcome in future studies.

In the studies of Li \& Yortsos (1995b) and Ioannis N. Tsimpanogiannis \& Yortsos (2002), considering the nucleation process in micromodels, it has been observed that the bubbles may grow after formation, while new bubbles are unlikely to be formed. This is a different picture 
of gas liberation than ours. It should be remarked that the concept of "bubble" in our paper is conditional. The number of bubbles is, simply, the measure of how much gas has appeared in a capillary. Since the geometry of a realistic capillary is random, this gas will appear randomly in different places. We assume that its appearance is totally random. This is, probably, an oversimplification. However, this is partly confirmed by the fact that, as discussed in Li \& Yortsos (1995b) and Yortsos \& Parlar (1989), the nucleation of the bubbles or droplets is heterogeneous. There are certain points around which the new phase is formed. One may assume that the points of growth of the bubbles or droplets are also randomly distributed. This is, probably, an inaccurate assumption for the regular pore network models where the experiments of Bora, Maini, \& Chakma (2000), El Yousfi, Zarcone, Bories, \& Lenormand(1997), Mackay, Henderson, Tehrani, Danesh (1998), and Nejad \& Danesh (2005) were carried out. However, for natural porous media, this assumption may hold.

An indirect confirmation of the validity of our model would be independence of the relative permeabilities and, in particular, of the critical saturations, on the dynamics of the process. It has been detected in the experiments of Firoozabadl et al. (1992), Jr, Agaev, \& Palatnik (2004), Li \& Yortsos (1995a), and Moulu (1989) that the critical saturation is dependent on the pressure decline rate. Such a dependence, obviously, contradicts our model. On the other hand, in the experiments analyzed in section 3, the relative permeabilities were found to be functions of the saturation only. In experimental works of Bagudu et al. (2018) and Kamath \& Boyer (1995), it has also be found that the critical gas saturations are independent or almost independent of the pressure decline rate and the nucleation fraction. These observations are valid for the lowpermeable natural porous media (Bagudu et al., 2018). Our model is obviously valid for the latter quasi-equilibrium liberation process, while a less equilibrium situation described in(Du \& Yortsos, 1999; X. Li \& Yortsos, 1994, 1995b; Xuehai Li \& Yortsos, 1993; Satik \& Yortsos, 1996; I N Tsimpanogiannis et al., 1999; Ioannis N. Tsimpanogiannis \& Yortsos, 2004; Yortsos \& Parlar, 1989 and others) cannot be described by this model. In particular, one might assume that the proposed model may somehow describe the behavior of the foamy oils. However, this behavior is very complex and rate-dependent (B. Maini, 1996; B. B. Maini, 1999; Smith, 1988), so that application of the model here requires much care.

Finally, we apply very simplified assumptions about conductivities of separate capillaries for the gas and liquid flow. The picture of such flow may be rather complicated (see e.g. (Wang, Andersen, \& Shapiro, 2020). Considering more detail is outside the scope of the present paper.

\section{Conclusions}

A new model for relative permeabilities under gas liberation or condensate precipitation in porous media has been developed. It is based on the methods of statistical physics and percolation theory. The porous medium is modeled as a capillary lattice. The gas liberation occurs in the form of separate bubbles, while oil precipitates in the form of the droplets. Distribution of the bubbles or the droplets in the capillaries is computed by the methods of statistical physics. The conductivity of a lattice is computed by the theory of effective medium.

The theory makes it possible to evaluate the relative permeabilities based on a relatively small number of the parameters, like the coordination number of the lattice or the critical fraction of the bubbles. A simple universal correlation between these parameters and the critical gas saturation is established. 
We show that the simple power dependencies (the Corey-Brooks model) may be used for fitting the physically meaningful dependencies derived in the framework of our model with a reasonable (although not absolute) accuracy. The comparison makes it possible to spread the Corey-Brooks dependencies outside the regions where both phases are mobile.

Comparison with available experimental data shows a good fit between the data and the model. Only the value of the critical bubble fraction needs to be fitted. For the cores of the same origin, this value varies insignificantly, which shows that the model captures, basically, the physics of the process.

\section{Acknowledgments}

This study has been performed in the framework of a project supported by the Danish Hydrocarbon Research and Technology Center (DHRTC, Technical University of Denmark). Dr. Charlotte Lassen is kindly acknowledged for multiple useful discussions of this work. Duc Thuong Vu and Tran Tsuong Dang are kindly acknowledged for invaluable help in the laboratory.

\section{References}

Afidick, D., Kaczorowski, N. J., \& Bette, S. (1994). Production Performance of a Retrograde Gas Reservoir: A Case Study of the Arun Field. In SPE Asia Pacific Oil and Gas Conference. Society of Petroleum Engineers. https://doi.org/10.2118/28749-MS

Bagudu, U., McDougall, S. R., \& Mackay, E. J. (2018). Network modelling analysis of a depressurization experiment on a North Sea reservoir core sample. Journal of Petroleum Science and Engineering, 162(June 2017), 63-75. https://doi.org/10.1016/j.petrol.2017.11.036

Bedrikovetsky, P. (1993). Mathematical Theory of Oil and Gas Recovery : With Applications to ex-USSR Oil and Gas Fields. (G. Rowan, Ed.) (1st ed., Vol. 4). Dordrecht: Springer Netherlands. https://doi.org/10.1007/978-94-017-2205-6

Behrenbruch, P., Hoang, T. G., Do Huu, M. T., Bui, K. D., \& Kennaird, T. (2018). The importance of optimal choice of relative permeability relationships in reservoir simulation. In Society of Petroleum Engineers - SPE Asia Pacific Oil and Gas Conference and Exhibition 2018, APOGCE 2018. Society of Petroleum Engineers. https://doi.org/10.2118/192109-ms

Blunt, M., \& King, P. (1991). Relative permeabilities from two- and three-dimensional porescale network modelling. Transport in Porous Media, 6(4), 407-433. https://doi.org/10.1007/BF00136349

Bora, R., Maini, B. B., \& Chakma, A. (2000). Flow visualization studies of solution gas drive process in heavy oil reservoirs with a glass micromodel. In SPE Reservoir Evaluation and Engineering (Vol. 3, pp. 224-229). Society of Petroleum Engineers. https://doi.org/10.2118/64226-pa

Brooks, R. H., \& Corey, A. T. (1964). Hydraulic properties of porous media. Fort Collins: Colorado State University [Hydrology and Water Resources Program].

Chatzis, I., Morrow, N. R., \& Lim, H. T. (1983). Magnitude and Detailed Structure of Residual Oil Saturation. Society of Petroleum Engineers Journal, 23(2), 311-326. https://doi.org/10.2118/10681-PA 
Chen, H. L., Wilson, S. D., \& Monger-McClure, T. G. (1999). Determination of relative permeability and recovery for North Sea gas-condensate reservoirs. SPE Reservoir Evaluation and Engineering, 2(4), 393-402. https://doi.org/10.2118/57596-PA

Chierici, G. L. (1984). NOVEL RELATIONS FOR DRAINAGE AND IMBIBITION RELATIVE PERMEABILITIES. Society of Petroleum Engineers Journal, 24(3), 275-276. https://doi.org/10.2118/10165-PA

Corey, A. T. (1977). Mechanics of heterogeneous fluids in porous media. Mechanics of heterogeneous fluids in porous media. Water Resources Publications. Retrieved from https://www.cabdirect.org/cabdirect/abstract/19801953411

Corey, A. T., \& Rathjens, C. H. (1956). Effect of Stratification on Relative Permeability. Journal of Petroleum Technology, 8(12), 69-71. https://doi.org/10.2118/744-g

Dake, L. P. (1994). The practice of reservoir engineering. Elsevier.

Du, C., \& Yortsos, Y. C. (1999). A numerical study of the critical gas saturation in a porous medium. Transport in Porous Media, 35(2), 205-225. https://doi.org/10.1023/A:1006582222356

El Yousfi, A., Zarcone, C., Bories, S., \& Lenormand, R. (1997). Physical mechanisms for bubble growth during solution gas drive. Proceedings - SPE Annual Technical Conference and Exhibition. https://doi.org/10.2523/38921-ms

Firoozabadi, A., \& Kashchiev, D. (1996). Pressure and volume evolution during gas phase formation in solution gas drive process. SPE Journal, 1(3), 219-226. https://doi.org/10.2118/26286-pa

Firoozabadl, A., Ottesen, B., \& Mikkelsen, M. (1992). Measurements of supersaturation and critical gas saturation. SPE Formation Evaluation, 7(4), 337-344. https://doi.org/10.2118/19694-pa

Ghanbarian, B., Hunt, A. G., Skinner, T. E., \& Ewing, R. P. (2015). Saturation dependence of transport in porous media predicted by percolation and effective medium theories. Fractals, 23(1). https://doi.org/10.1142/S0218348X15400046

Gravier, J. F., Lemouzy, P., Barroux, C., \& Abed, A. F. (1986). Determination of Gas-Condensate Relative Permeability on Whole Cores Under Reservoir Conditions. SPE Formation Evaluation, 1(01), 9-15. https://doi.org/10.2118/11493-PA

Honerkamp, J. (2012). Statistical physics : an advanced approach with applications. Springer.

Hunt, A., Ewing, R., \& Ghanbarian, B. (2014). Percolation Theory for Flow in Porous Media (Vol. 880). Cham: Springer International Publishing. https://doi.org/10.1007/978-3-31903771-4

Jr, E. P., Agaev, G., \& Palatnik, B. (2004). Determination of critical gas saturation and relative permeabilities relevant to the depressurization of the Statfjord Field. Symposium of The, (December), 1-15.

Kalla, S., Leonardi, S. A., Berry, D. W., Poore, L. D., Sahoo, H., Kudva, R. A., \& Braun, E. (2014). Factors That Affect Gas-Condensate Relative Permeability. SPE Reservoir Evaluation \& 
Engineering, Preprint(Preprint), 1-9. https://doi.org/10.2118/173177-PA

Kamath, J., \& Boyer, R. E. (1995). Critical gas saturation and supersaturation in lowpermeability rocks. SPE Formation Evaluation, 10(4), 247-253. https://doi.org/10.2118/26663-pa

Kashchiev, D., \& Firoozabadi, A. (1993). Kinetics of the initial stage of isothermal gas phase formation. The Journal of Chemical Physics, 98(6), 4690-4699. https://doi.org/10.1063/1.465065

Kirkpatrick, S. (1973a). Percolation ancl Conduction, 45.

Kirkpatrick, S. (1973b). Percolation and Conduction. Reviews of Modern Physics, 45(4), 574588. https://doi.org/10.1103/RevModPhys.45.574

Kubo, R. (1965). Statistical Mechanics. (H. Ichimura, T. Usui, \& N. (Natsuki) Hashitsume, Eds.), Physics Today (Vol. 18). North Holland. https://doi.org/10.1063/1.3046964

Landau, L. D., \& Lifshits, E. M. (1980). Statistical Physics. Elsevier. https://doi.org/10.1016/C2009-0-24487-4

Li, X., \& Yortsos, Y. C. (1994). Bubble growth and stability in an effective porous medium. Physics of Fluids, 6(5), 1663-1676. https://doi.org/10.1063/1.868229

$\mathrm{Li}, \mathrm{X} .$, \& Yortsos, Y. C. (1995a). Theory of multiple bubble growth in porous media by solute diffusion. Chemical Engineering Science, 50(8), 1247-1271. https://doi.org/10.1016/0009-2509(95)98839-7

Li, X., \& Yortsos, Y. C. (1995b). Visualization and simulation of bubble growth in pore networks. AIChE Journal, 41(2), 214-222. https://doi.org/10.1002/aic.690410203

Li, Xuehai, \& Yortsos, Y. C. (1993). Critical gas saturation: modeling and sensitivity studies. Proceedings - SPE Annual Technical Conference and Exhibition, Sigma, 589-604. https://doi.org/10.2523/26662-ms

Mackay, E. J., Henderson, G. D., Tehrani, D. H., Danesh, A., \& Herlot-Watt, U. (1998). The Importance of Interfacial Tension on Fluid Distribution during Depressurization. SPE Reservoir Engineering (Society of Petroleum Engineers), 1(5), 408-415. https://doi.org/10.2118/51761-pa

Maini, B. (1996). Foamy Oil Flow In Heavy Oil Prodution. Journal of Canadian Petroleum Technology, 35(06). https://doi.org/10.2118/96-06-01

Maini, B. B. (1999). Foamy Oil Flow in Primary Production of Heavy Oil under Solution Gas Drive. Society of Petroleum Engineers (SPE). https://doi.org/10.2118/56541-ms

Mogensen, K., \& Stenby, E. H. (1998). A Dynamic Two-Phase Pore-Scale Model of Imbibition. Transport in Porous Media, 32(1-3), 299-327. https://doi.org/10.2118/39658-ms

Moulu, J. C. (1989). Solution-gas drive: experiments and simulation. Journal of Petroleum Science and Engineering, 2(4), 379-386. https://doi.org/10.1016/0920-4105(89)900119

Nejad, K. S., \& Danesh, A. (2005). Visual Investigation of Oil Depressurisation in Pores with 
Different Wettability Characteristics and Saturation Histories (SPE94054). In 67th EAGE Conference \& Exhibition (p. cp-1-00278). European Association of Geoscientists \& Engineers. https://doi.org/10.3997/2214-4609-pdb.1.D035

Patsoules, M. G., \& Cripps, J. C. (1983). A quantitative analysis of chalk pore geometry using resin casts. Energy Sources, 7(1), 15-31. https://doi.org/10.1080/00908318308908072

Pope, G. A., Wu, W., Narayanaswamy, G., Delshad, M., Sharma, M., \& Wang, P. (1998). Modeling relative permeability effects in gas-condensate reservoirs. Proceedings - SPE Annual Technical Conference and Exhibition, 1999-Septe, 643-654. https://doi.org/10.2523/49266-ms

Prat, M. (2002). Recent advances in pore-scale models for drying of porous media. Chemical Engineering Journal (Vol. 86).

Sahimi, M. (2011). Flow and Transport in Porous Media and Fractured Rock. Weinheim, Germany: Wiley-VCH Verlag GmbH \& Co. KGaA. https://doi.org/10.1002/9783527636693

Satik, C., \& Yortsos, Y. C. (1996). A pore-network study of bubble growth in porous media driven by heat transfer. Journal of Heat Transfer, 118(2), 455-462. https://doi.org/10.1115/1.2825866

Selyakov, V., \& Kadet, V. (1996). Percolation models for transport in porous media with applications to reservoir engineering. Kluwer Academic Publishers.

Smith, G. E. (1988). FLUID FLOW AND SAND PRODUCTION IN HEAVY-OIL RESERVOIRS UNDER SOLUTION-GAS DRIVE. SPE Production Engineering, 3(2), 169-180. https://doi.org/10.2118/15094-PA

Tsimpanogiannis, I N, Yortsos, Y. C., Poulou, S., Kanellopoulos, N., \& Stubos, A. K. (1999). Scaling theory of drying in porous media. The American Physical Society, 59(4).

Tsimpanogiannis, loannis N., \& Yortsos, Y. C. (2002). Model for the gas evolution in a porous medium driven by solute diffusion. AIChE Journal, 48(11), 2690-2710. https://doi.org/10.1002/aic.690481126

Tsimpanogiannis, loannis N., \& Yortsos, Y. C. (2004). The critical gas saturation in a porous medium in the presence of gravity. Journal of Colloid and Interface Science, 270(2), 388395. https://doi.org/10.1016/j.jcis.2003.09.036

Wang, T., Andersen, S. I., \& Shapiro, A. (2020). Coalescence of oil droplets in microchannels under brine flow. Colloids and Surfaces A: Physicochemical and Engineering Aspects, 598, 124864. https://doi.org/10.1016/j.colsurfa.2020.124864

Woo, M. W. (2019). Heat and mass transfer in drying of porous media. Drying Technology, 12. https://doi.org/10.1080/07373937.2019.1686518

Yortsos, Y. C., \& Parlar, M. (1989). Phase change in binary systems in porous media: application to solution-gas drive. Society of Petroleum Engineers of AIME, (Paper) SPE, GAMMA. https://doi.org/10.2118/19697-ms 